TITLE:

\title{
Efficient implementation of three- dimensional reference interaction site model self-consistent-field method: Application to solvatochromic shift calculations
}

$\operatorname{AUTHOR}(S)$ :

Minezawa, N; Kato, S

\section{CITATION:}

Minezawa, N ... [et al]. Efficient implementation of three-dimensional reference interaction site model self-consistent-field method: Application to solvatochromic shift calculations. JOURNAL OF CHEMICAL PHYSICS 2007, 126(5): 054511.

ISSUE DATE:

2007-02-07

URL:

http://hdl.handle.net/2433/50058

\section{RIGHT:}

Copyright 2007 American Institute of Physics. This article may be downloaded for personal use only. Any other use requires prior permission of the author and the American Institute of Physics. 


\title{
Efficient implementation of three-dimensional reference interaction site model self-consistent-field method: Application to solvatochromic shift calculations
}

\author{
Noriyuki Minezawa and Shigeki Kato ${ }^{a)}$ \\ Department of Chemistry, Graduate School of Science, Kyoto University, Kitashirakawa, Sakyo-ku, \\ Kyoto 606-8502, Japan
}

(Received 2 November 2006; accepted 15 December 2006; published online 6 February 2007)

\begin{abstract}
The authors present an implementation of the three-dimensional reference interaction site model self-consistent-field (3D-RISM-SCF) method. First, they introduce a robust and efficient algorithm for solving the 3D-RISM equation. The algorithm is a hybrid of the Newton-Raphson and Picard methods. The Jacobian matrix is analytically expressed in a computationally useful form. Second, they discuss the solute-solvent electrostatic interaction. For the solute to solvent route, the electrostatic potential (ESP) map on a 3D grid is constructed directly from the electron density. The charge fitting procedure is not required to determine the ESP. For the solvent to solute route, the ESP acting on the solute molecule is derived from the solvent charge distribution obtained by solving the 3D-RISM equation. Matrix elements of the solute-solvent interaction are evaluated by the direct numerical integration. A remarkable reduction in the computational time is observed in both routes. Finally, the authors implement the first derivatives of the free energy with respect to the solute nuclear coordinates. They apply the present method to "solute" water and formaldehyde in aqueous solvent using the simple point charge model, and the results are compared with those from other methods: the six-dimensional molecular Ornstein-Zernike SCF, the one-dimensional site-site RISM-SCF, and the polarizable continuum model. The authors also calculate the solvatochromic shifts of acetone, benzonitrile, and nitrobenzene using the present method and compare them with the experimental and other theoretical results. (C) 2007 American Institute of Physics.
\end{abstract}

[DOI: $10.1063 / 1.2431809]$

\section{INTRODUCTION}

The development of quantum chemical methods to describe the structural and thermodynamic properties of molecules in solution is of crucial importance for investigating chemical and biological processes. ${ }^{1}$ Based on the dielectric continuum model of solvent, several methods have been developed to calculate the electronic structure of solvated molecules. ${ }^{2-4}$ In these approaches, the solvation effects are directly incorporated in solute electronic structure calculations by solving the classical electromagnetic equations for solvent response. Although these models have been applied successfully to various chemical processes in solution, they have a difficulty in describing a local solute-solvent interaction such as hydrogen bonding since the solvent is characterized by a macroscopic parameter, i.e., the dielectric constant.

In order to take account of the microscopic aspects of solvent molecules, the integral equation theories of polyatomic molecular liquids have been employed to describe the solute-solvent interaction. ${ }^{5}$ The molecular Ornstein-Zernike (MOZ) approach ${ }^{6-10}$ and its combination with ab initio calculations of solute molecules ${ }^{11}$ have been proposed. In the MOZ theory, the orientational dependence of intermolecular interactions is treated through the rotational invariant expansions of correlation functions as well as the interaction po-

${ }^{a)}$ Electronic mail: shigeki@kuchem.kyoto-u.ac.jp tentials by generalized spherical harmonics. Although the equations are easily solved for the solute whose shape is spherical or nearly spherical, it is difficult to apply such a method to highly nonspherical molecules because the multipole expansions converge slowly.

In the last decade, a theoretical model, referred to as the reference interaction site model self-consistent-field (RISM$\mathrm{SCF})$ method, ${ }^{12-14}$ has been developed. In the RISM theory, the molecular aspects of the solvent are incorporated by employing the site-site $\mathrm{OZ}$ equation obtained by averaging both the solute and solvent orientations. The solute-solvent interaction is thus represented approximately as the sum of pair potentials which depend only on the site-site distances. Hereafter, the site-site RISM-SCF method is denoted as the onedimensional RISM-SCF (1D-RISM-SCF) method.

In order to obtain more detailed information on the solvation structure, a three-dimensional (3D) reduction of the six-dimensional (6D) OZ equation has been devised. ${ }^{15-21} \mathrm{Be}-$ glov and Roux solved the hypernetted chain (HNC) integral equation on a 3D grid and applied the method to a system composed of a solute with an irregular shape in a liquid of spherical particles. ${ }^{15}$ Later, they extended this approach to a liquid of spheres with an embedded dipole and formulated the three-dimensional mean spherical approximation integral equation. ${ }^{16}$ Ikeguchi and Doi employed spherical solvents and solved the original RISM equation directly on a 3D grid. ${ }^{17}$ Cortis et al. derived a 3D integral equation for solute 
molecule-solvent site correlation functions and applied the equation to one-component molecular liquids. ${ }^{18}$ Kovalenko ${ }^{19}$ and Kovalenko and Hirata ${ }^{20,21}$ developed the 3D generalization of the RISM (3D-RISM) method to obtain the threedimensional distributions of solvent sites around the solute.

Although the 3D-RISM-SCF method has advantages over the 1D-RISM-SCF, its applications to polyatomic solute systems are still limited ${ }^{21-24}$ because of the computational difficulties. Sato et al. proposed a SCF combination of the $a b$ initio molecular orbital theory and 3D-RISM approach, ${ }^{22}$ and applied the method to a simple diatomic molecule. Du and Wei applied the 3D-RISM-SCF procedure to $N$-methylacetamide. $^{23}$ But they approximately constructed the electrostatic potential (ESP) map by using the fitted atomic charges to obtain the solvent distribution. This is because it is much more difficult to treat the solute-solvent interaction on a 3D grid than on a radial 1D grid employed in the site-site RISM theory. However, it is not sufficient to describe the ESP based on the superposition of Coulomb potentials generated by discretized partial charges. For example, the directionality of the ESP due to solute lone pair orbitals cannot be expressed as the sum of atomic charges. Very recently, Yoshida and Hirata have proposed a hybrid approach, where the ESP is constructed by explicitly calculating the solute electron density at the short-range region and by using fitted charges at the distant region. ${ }^{24}$

In the present paper, we describe a new implementation of the 3D-RISM-SCF method. First, we develop a robust and efficient algorithm for solving the 3D-RISM equation. Several methods ${ }^{21,25-28}$ have been devised to achieve the fast convergence. The algorithm adopted here is a hybrid of the Newton-Raphson (NR) and Picard (PC) methods, ${ }^{29-32}$ which is successfully applied to the site-site RISM equation. The Jacobian matrix is given analytically and expressed in a computationally useful form. Second, we discuss efficient and accurate calculations of the solute-solvent electrostatic interaction. For the solute to solvent route, i.e., the ESP acting on the solvent, the ESP map on a 3D grid is constructed directly from the electron density. It is not necessary to approximate the ESP as the sum of Coulomb potentials generated by the fitted partial charges on solute sites. For the solvent to solute route, i.e., the ESP acting on the solute, the matrix elements of the solute-solvent interaction are evaluated by the direct numerical integration with the ESP derived from the solvent charge distribution. Finally, the analytical free energy gradient with respect to solute nuclear coordinates is implemented. We apply the present method to water and formaldehyde molecules in aqueous solution to compare the results with those from other methods: the 6D MOZ-SCF, 1DRISM-SCF, and polarizable continuum model (PCM). The solvatochromic shifts of vertical excitation energies for acetone, benzonitrile, and nitrobenzene in aqueous solution are also calculated, and the results are compared with the experiments. In these calculations, we used the simple point charge (SPC)-like model $^{33}$ to describe the water solvent.

The organization of this paper is as follows. In Sec. II, we describe the present implementation of the 3D-RISMSCF method. The calculation methods of the solute-solvent electrostatic interaction are discussed. We also derive the analytical free energy gradients. In Sec. III, we present the calculated results. The timing and accuracy of the present implementation are discussed. Concluding remarks are summarized in Sec. IV.

\section{THEORETICAL METHODS}

\section{A. Formalism and numerical methods}

Since the derivation and details of the 3D-RISM equation have been discussed thoroughly in Refs. 19-21, we only briefly summarize the theoretical methods applied here. The 3D solute-solvent RISM equation at infinite dilution is given by

$$
\begin{aligned}
& \tilde{\eta}_{\alpha}(\mathbf{k})=\tilde{c}_{\mu}(\mathbf{k}) \tilde{H}_{\mu \alpha}^{V V}(k)-\tilde{c}_{\alpha}(\mathbf{k}), \\
& \tilde{\eta}_{\alpha}(\mathbf{k})=\tilde{h}_{\alpha}(\mathbf{k})-\tilde{c}_{\alpha}(\mathbf{k}),
\end{aligned}
$$

and

$$
\tilde{H}_{\mu \alpha}^{V V}(k)=\widetilde{\omega}_{\mu \alpha}(k)+\rho \tilde{h}_{\mu \alpha}^{V V}(k),
$$

where the tilde denotes the quantity in reciprocal space and the summation over repeating indices of solvent sites, $\mu$ and $\alpha$, is implied. Here $c$ and $h$ are the direct and total correlation functions, respectively. $\omega$ is the intramolecular correlation function and $\rho$ is the number density of the solvent. The solvent correlation function $\widetilde{h}_{\mu \alpha}^{V V}$ is obtained by solving the solvent-solvent RISM equation,

$$
\tilde{h}_{\mu \alpha}^{V V}(k)=\widetilde{\omega}_{\mu \nu}^{V}(k) \tilde{c}_{\nu \gamma}^{V V}(k)\left[\tilde{\omega}_{\gamma \alpha}^{V}(k)+\rho \tilde{h}_{\gamma \alpha}^{V V}(k)\right],
$$

and is saved in reciprocal space. They are interpolated in reciprocal space at the absolute value $k$ corresponding to the 3D mesh point $\mathbf{k}$.

In the present calculations, we use the $3 \mathrm{D}$ generalization of the HNC closure (3D-HNC),

$$
c_{\alpha}(\mathbf{r})=\exp \left[-\beta u_{\alpha}(\mathbf{r})+\eta_{\alpha}(\mathbf{r})\right]-\eta_{\alpha}(\mathbf{r})-1,
$$

where $\beta=1 / k_{B} T$ is the inverse temperature and $u_{\alpha}$ is the interaction potential between the solute molecule and the solvent site $\alpha$.

Care must be taken in the treatment of the long-range Coulomb potential. It is convenient to define the short-range parts of direct correlation functions and interaction potentials by

$$
\begin{aligned}
& c_{\alpha}^{s}(\mathbf{r})=c_{\alpha}(\mathbf{r})-\phi_{\alpha}^{l}(\mathbf{r}), \\
& \phi_{\alpha}^{*}(\mathbf{r})=-\beta u_{\alpha}(\mathbf{r})-\phi_{\alpha}^{l}(\mathbf{r}),
\end{aligned}
$$

and

$$
\phi_{\alpha}^{l}(\mathbf{r})=-\beta q_{\alpha} \sum_{b} \frac{Q_{b} \operatorname{erf}\left(\lambda\left|\mathbf{r}-\mathbf{R}_{b}\right|\right)}{\left|\mathbf{r}-\mathbf{R}_{b}\right|},
$$

where $Q_{b}$ and $q_{\alpha}$ are the solute and solvent partial charges, respectively, and erf denotes the error function. The solute charges are determined by the least-squares fitting. The accuracy of the fitting procedure is not so critical because it is sufficient to reproduce the long-range potential by using the fitted charges. Note that these charges are not used for the 
construction of ESP. The parameter $\lambda$ is set to be $1.13 \AA^{-1}$ throughout the calculations. The Fourier transform of Eq. (4) gives

$$
\begin{aligned}
& \widetilde{c}_{\alpha}^{s}(\mathbf{k})=\widetilde{c}_{\alpha}(\mathbf{k})-\widetilde{\phi}_{\alpha}^{l}(\mathbf{k}) \\
& \widetilde{\phi}_{\alpha}^{l}(\mathbf{k})=-4 \pi \beta q_{\alpha} \sum_{b} \frac{Q_{b}}{k^{2}} e^{i \mathbf{k} \cdot \mathbf{R}_{b}-k^{2} / 4 \lambda^{2}}
\end{aligned}
$$

Based on Eqs. (4) and (5), we convert the 3D-RISM equation [Eq. (1a)] and the 3D-HNC closure in Eq. (3) to a renormalized form involving the short-range correlation functions $\theta_{\alpha}$ and $c_{\alpha}^{s}$ as

$$
\begin{aligned}
\tilde{\theta}_{\alpha}(\mathbf{k})= & -\widetilde{c}_{\alpha}^{s}(\mathbf{k})+\widetilde{c}_{\mu}^{s}(\mathbf{k}) \tilde{H}_{\mu \alpha}^{V V}(k), \\
c_{\alpha}^{s}(\mathbf{r})= & \exp \left[\gamma_{\alpha}(\mathbf{r})+\phi_{\alpha}^{*}(\mathbf{r})\right] \\
& \times \exp \left[\theta_{\alpha}(\mathbf{r})\right]-\gamma_{\alpha}(\mathbf{r})-\theta_{\alpha}(\mathbf{r})-1,
\end{aligned}
$$

where

$$
\begin{aligned}
& \tilde{\gamma}_{\alpha}(\mathbf{k})=\widetilde{\phi}_{\mu}^{l}(\mathbf{k}) \tilde{H}_{\mu \alpha}^{V V}(k), \\
& \tilde{\theta}_{\alpha}(\mathbf{k})=\tilde{h}_{\alpha}(\mathbf{k})-\widetilde{c}_{\alpha}^{s}(\mathbf{k})-\widetilde{\gamma}_{\alpha}(\mathbf{k}) .
\end{aligned}
$$

Here the functions $\theta_{\alpha}$ and $c_{\alpha}^{s}$ are determined iteratively. Each iteration cycle involves the calculations of forward and backward three-dimensional fast Fourier transform (3D-FFT). In the present formulation, however, the error arising from the periodicity of Fourier transform is negligible because $\theta_{\alpha}$ and $c_{\alpha}^{s}$ are short-range functions compared with the system size.

The hybrid NR/PC method is applied to the renormalized form above. Each $\theta_{\alpha}(\mathbf{r})$ is decomposed into the "coarse" and "fine" parts according to the scheme proposed by Gillan. ${ }^{34}$ The former is expressed in terms of the basis functions, i.e., roof functions. $\theta_{\alpha}$ can be written as

$$
\theta_{\alpha}(\mathbf{r})=\sum_{s} a_{\alpha s} P_{s}(\mathbf{r})+\delta \theta_{\alpha}(\mathbf{r})
$$

where $P_{s}$ is the $s$ th basis function defined in the Appendix. The fine part $\delta \theta_{\alpha}$ is orthogonal to the basis functions,

$$
\sum_{\mathbf{r}} P_{s}(\mathbf{r}) \delta \theta_{\alpha}(\mathbf{r})=0 .
$$

The new coefficient $a_{\alpha s}^{\text {new }}$ is prepared by the NR procedure using the input $a_{\alpha s}^{\text {old }}$ and the resultant output $a_{\alpha s}^{\prime}$ in the previous step,

$$
a_{\alpha s}^{\text {new }}=a_{\alpha s}^{\mathrm{old}}-\sum_{\mu t}\left(J^{-1}\right)_{\alpha s, \mu t}\left(a_{\mu t}^{\mathrm{old}}-a_{\mu t}^{\prime}\right) .
$$

It is straightforward to derive the analytical expression for the Jacobian matrix $J$ within the framework of the 3D-RISM theory. Details of the derivation are given in the Appendix. Since the Jacobian matrix elements depend on $\theta_{\alpha}$ in the present formulation, the update is required during the iterations. However, we construct the Jacobian matrix only once at the beginning of the 3D-RISM-SCF cycle. The same matrix is used for solving the 3D-RISM equations until the 3D-RISM-SCF is converged. This is partly because much computational time is involved in calculating the Jacobian matrix. In the site-site RISM case, it is suggested that the same matrix can be reused even for different conformations of the solute molecule. ${ }^{29}$

\section{B. Electrostatic interaction}

\section{Solute to solvent}

We assume the solute-solvent interaction potential of the form

$$
u_{\alpha}(\mathbf{r})=u_{\alpha}^{\text {short }}(\mathbf{r})+u_{\alpha}^{\mathrm{es}}(\mathbf{r})
$$

Here the first term, the short-range potential, is represented by the site-site Lennard-Jones (LJ) potentials,

$$
u_{\alpha}^{\text {short }}(\mathbf{r})=\sum_{b} 4 \epsilon_{b \alpha}\left\{\left(\frac{\sigma_{b \alpha}}{\left|\mathbf{r}-\mathbf{R}_{b}\right|}\right)^{12}-\left(\frac{\sigma_{b \alpha}}{\left|\mathbf{r}-\mathbf{R}_{b}\right|}\right)^{6}\right\} .
$$

The electrostatic contribution is given by

$$
u_{\alpha}^{\mathrm{es}}(\mathbf{r})=q_{\alpha}\left\{\sum_{b} \frac{Z_{b}}{\left|\mathbf{r}-\mathbf{R}_{b}\right|}-\sum_{p q} D_{p q} A_{p q}(\mathbf{r})\right\},
$$

where $A_{p q}$ is a three-center one electron integral in terms of atomic basis functions $\chi_{p}$ and $\chi_{q}$,

$$
A_{p q}(\mathbf{r})=\int d \mathbf{r}^{\prime} \chi_{p}\left(\mathbf{r}^{\prime}\right) \frac{1}{\left|\mathbf{r}-\mathbf{r}^{\prime}\right|} \chi_{q}\left(\mathbf{r}^{\prime}\right)
$$

and $D_{p q}$ is a density matrix element. The calculation of ESP by Eq. (14) takes much computational time because it requires the integrals at $N^{3}$ (e.g., $N=128$ ) points on the $3 \mathrm{D}$ grid and the cost also depends on the number of atomic basis functions.

Sato et al. proposed a method to save computational time. $^{22}$ The ESP from solute nuclei was obtained by the Ewald summation within the supercell method. To evaluate the contribution from the solute electrons, they constructed the electron density in reciprocal space analytically and solved the reciprocal space Poisson equation. The ESP map can be easily obtained by the backward 3D-FFT.

We employ a similar approach so that the resultant ESP map reproduces Eq. (14). It is noted that the present formulation treats not an infinitely replicated periodic system but a nonperiodic cluster. The nuclear contribution to the ESP is expressed as the sum of bare Coulomb potentials.

The ESP originated from the electron distribution is simply written by the Coulomb law in real space as

$$
\psi(\mathbf{r})=-\int_{V} d \mathbf{r}^{\prime} n\left(\mathbf{r}^{\prime}\right) f\left(\mathbf{r}-\mathbf{r}^{\prime}\right),
$$

where $n(\mathbf{r})$ is the electron density and $f(\mathbf{r})=1 /|\mathbf{r}|$. Using the plane-wave expansion $^{35}$ of $n(\mathbf{r})$ and $f(\mathbf{r})$, the ESP is expressed by

$$
\psi(\mathbf{r})=-\frac{1}{V} \sum_{\mathbf{k}} \bar{n}(-\mathbf{k}) \bar{f}(\mathbf{k}) e^{-i \mathbf{k} \cdot \mathbf{r}}
$$

It is difficult to evaluate $\bar{f}(\mathbf{k})$, the Fourier expansion coefficients of the bare Coulomb potential, by the 3D-FFT because of the singularity at $\mathbf{r}=0$. We employ the reciprocal space method proposed by Martyna and Tuckerman, ${ }^{36}$ which gives 
accurate and numerically efficient descriptions of long-range interaction. As in the case of the Ewald method, the Coulomb potential can be written as

$$
f(\mathbf{r}) \equiv \frac{1}{r}=\frac{\operatorname{erf}(\alpha r)}{r}+\frac{\operatorname{erfc}(\alpha r)}{r} \equiv f^{l}(\mathbf{r})+f^{s}(\mathbf{r}),
$$

where erfc denotes the complementary error function. Since $f^{s}(\mathbf{r})$ decays rapidly at longer distance in real space, it is possible to write

$$
\bar{f}^{s}(\mathbf{k})=\int_{V} d \mathbf{r} f^{s}(\mathbf{r}) e^{i \mathbf{k} \cdot \mathbf{r}} \simeq \int_{\text {all space }} d \mathbf{r} f^{s}(\mathbf{r}) e^{i \mathbf{k} \cdot \mathbf{r}}=\widetilde{f}^{s}(\mathbf{k}) .
$$

From Eq. (18), the Fourier coefficients $\bar{f}(\mathbf{k})$ are given by

$$
\bar{f}(\mathbf{k})=\bar{f}^{s}(\mathbf{k})+\bar{f}^{\prime}(\mathbf{k}) \simeq \tilde{f}^{s}(\mathbf{k})+\bar{f}^{l}(\mathbf{k})=\tilde{f}(\mathbf{k})+\bar{f}^{l}(\mathbf{k})-\tilde{f}^{l}(\mathbf{k}) .
$$

Inserting this approximation to Eq. (17), the ESP is given by

$$
\psi(\mathbf{r})=-\frac{1}{V} \sum_{\mathbf{k}} \bar{n}(-\mathbf{k})\left[\widetilde{f}(\mathbf{k})+\left\{\bar{f}^{l}(\mathbf{k})-\widetilde{f}^{l}(\mathbf{k})\right\}\right] e^{-i \mathbf{k} \cdot \mathbf{r}} .
$$

We can evaluate $\bar{f}(\mathbf{k})$ and $\bar{f}^{l}(\mathbf{k})$ analytically, and obtain $\bar{f}^{l}(\mathbf{k})$ numerically by using the 3D-FFT of $f^{l}(\mathbf{r})$ because it has no singularity in real space. With this modification, we can treat the ESP of the nonperiodic cluster system by the Fourier transform method. Note that the divergent behavior at $\mathbf{k}=0$ is eliminated in Eq. (21).

There are two important problems in Eq. (21). First, it has been pointed out that in the application of Fourier transform based methods the box size should be large enough to avoid the interaction with the charge densities in the neighboring boxes. $^{36,37}$ But it was found that the additional cost due to a larger array of $\tilde{n}(\mathbf{k})$ is relatively low in the present computation (see Sec. III). Second, it is difficult to treat compact Gaussian basis functions for the preparation of $\tilde{n}(\mathbf{k})$ because of their slow convergence in reciprocal space. Thus we divided Gaussian functions into compact and diffuse types based on their Gaussian exponents. We employ the criteria for the maximum allowed exponent proposed by Füsti-Molnár and Pulay, ${ }^{38}$ which is proportional to the inverse square of the grid width and is weakly dependent on the angular momentum. With the basis functions separated into compact (c) and diffuse (d) types, the three-center one electron integrals in Eq. (15) are classified as (cc), (cd), and (dd). In the present application, only the last class is calculated by the Fourier transform based method. The other two classes are treated by the multipole expansion ${ }^{39}$ up to the second order. The direct application of the multipole expansion is very expensive because the computational cost scales linearly as the number of the pairs of primitive Gaussian functions. To reduce the computational costs, we prepared $\left(N_{\mathrm{div}}+1\right)$ equally spaced points for each atom-atom pair and moved the center of the product of two Gaussian functions to the nearest neighbor point. The multipoles are evaluated with respect to these origins. We adopt $N_{\mathrm{div}}=10$ throughout the calculations.

\section{Solvent to solute}

In order to describe the solute electronic polarization induced by solvents, the solvated Fock operator is constructed. It can be easily derived in the same way as for the 1D-RISMSCF method. ${ }^{14}$ The one electron operator is modified by adding the following solute-solvent interaction term:

$$
\begin{aligned}
h_{p q}^{\mathrm{es}} & \equiv\left\langle\chi_{p}\left|\sum_{\alpha} \int d \mathbf{r} \rho \hat{u}_{\alpha}^{\mathrm{es}}(\mathbf{r}) g_{\alpha}(\mathbf{r})\right| \chi_{q}\right\rangle \\
& =-\iint d \mathbf{r} d \mathbf{r}^{\prime} \chi_{p}\left(\mathbf{r}^{\prime}\right) \frac{q^{v}(\mathbf{r})}{\left|\mathbf{r}-\mathbf{r}^{\prime}\right|} \chi_{q}\left(\mathbf{r}^{\prime}\right) \\
& \equiv-\int d \mathbf{r} q^{v}(\mathbf{r}) A_{p q}(\mathbf{r})
\end{aligned}
$$

where $q^{v}(\mathbf{r})=\rho \Sigma_{\alpha} q_{\alpha} g_{\alpha}(\mathbf{r})$ is the charge distribution function $(\mathrm{CDF})$ obtained by solving the 3D-RISM equations. $g_{\alpha}(\mathbf{r})$ is the distribution function of the solvent site $\alpha$. The evaluation of $A_{p q}$ takes much computational cost as in the case of the solute to solvent route.

For the efficient evaluation of the Fock matrix, we first integrate over $\mathbf{r}$, which leads to the ESP acting on the solute,

$$
\begin{aligned}
\psi\left(\mathbf{r}^{\prime}\right) & =\int_{V} d \mathbf{r} \frac{q^{v}(\mathbf{r})}{\left|\mathbf{r}-\mathbf{r}^{\prime}\right|} \\
& =\sum_{\mathbf{k}} \bar{q}^{v}(-\mathbf{k})\left[\tilde{f}(\mathbf{k})+\bar{f}^{\prime}(\mathbf{k})-\widetilde{f}^{\prime}(\mathbf{k})\right] e^{-i \mathbf{k} \cdot \mathbf{r}^{\prime}}
\end{aligned}
$$

Here $\bar{q}^{v}(\mathbf{k})$ is prepared from the CDF by using the 3D-FFT. To prevent the interaction with the $\mathrm{CDF}$ in the neighboring boxes, the box size is increased and the CDF is embedded in the larger cell. After the multiplications in reciprocal space, $\psi\left(\mathbf{r}^{\prime}\right)$ can be obtained by the backward 3D-FFT. In the integration of Eq. (22) over $\mathbf{r}^{\prime}$, we use the 3D direct product of the Gauss-Hermite quadrature for the primitive Gaussian functions $g_{i p}$ and $g_{j q}$,

$$
\begin{aligned}
h_{p q}^{\mathrm{es}}= & -\sum_{i j} d_{i p} d_{j q} \int d \mathbf{r}^{\prime} g_{i p}\left(a_{i p}, \mathbf{r}^{\prime}-\mathbf{R}_{A}\right) \psi\left(\mathbf{r}^{\prime}\right) g_{j q}\left(a_{j q}, \mathbf{r}^{\prime}-\mathbf{R}_{B}\right) \\
= & -\sum_{i j} d_{i p} d_{j q} \exp \left(-\frac{a_{i p} a_{j q}}{a_{i p}+a_{j q}} R_{A B}^{2}\right) t^{-3} \\
& \left.\times \sum_{\mathbf{n}}^{\operatorname{zeros}} \psi\left(\frac{\mathbf{r}_{\mathbf{n}}}{t}+\mathbf{R}_{P}\right) \prod_{\alpha=x, y, z} w_{n_{\alpha}}\left[\left(\frac{\mathbf{r}_{\mathbf{n}}}{t}+\mathbf{R}_{P A}\right)_{\alpha}\right]\right]_{\alpha}^{p_{\alpha}} \\
& \times\left[\left(\frac{\mathbf{r}_{\mathbf{n}}}{t}+\mathbf{R}_{P B}\right)_{\alpha}\right]
\end{aligned}
$$

where $t=\left(a_{i p}+a_{j q}\right)^{1 / 2}$ and $\mathbf{R}_{P A}=\mathbf{R}_{P}-\mathbf{R}_{A} \cdot w_{n_{\alpha}}$ and $\left(\mathbf{r}_{\mathbf{n}}\right)_{\alpha}$ are the weights and zeros of Hermite polynomials along the $\alpha$ axis. In the quadrature, the values of ESP at arbitrary points are required. They are generated by the tricubic interpolation of $\psi(\mathbf{r})$, which is expressed as the cubic function of the [0:1]normalized coordinates of $x, y$, and $z$, 


$$
\psi\left(\mathbf{r}_{i}\right)=\sum_{l=0}^{3} \sum_{m=0}^{3} \sum_{n=0}^{3} A_{l m n}^{(i)} x^{l} y^{m} z^{n}
$$

where the coefficients $A_{l m n}^{(i)}$ are determined by the values of $\psi, \nabla \psi, \psi_{x y}, \psi_{y z}, \psi_{z x}$, and $\psi_{x y z}$ at the eight nearest points surrounding $\mathbf{r}_{i}$. The derivatives of $\psi$ can be easily obtained simply by differentiating Eq. (23). It is essential to use the tricubic interpolation because of the following properties: (1) the values of the function and the specified derivatives are reproduced exactly on each mesh point, and (2) the gradient of the interpolated function changes continuously at the boundaries of each grid cube. In the case of the trilinear interpolation, for example, the derivative of the interpolated function changes discontinuously as the interpolating point crosses from one grid cube to another.

To be consistent with the multipole expansion employed in the solute to solvent route, we used the multipole expansion method to evaluate the matrix elements resulting from (cc) and (cd) types. Note that the multipoles and the ESP are evaluated at $N_{\text {div }}+1$ points for each atom-atom pair which are previously determined in the solute to solvent route.

\section{Energy gradient}

The free energy gradient calculations require the derivative of the average solute-solvent interaction with respect to the nuclear coordinates,

$$
\begin{aligned}
\frac{\partial}{\partial R_{c}} \sum_{\alpha}\left\langle u_{\alpha}\right\rangle= & \sum_{\alpha}\left\langle\frac{\partial u_{\alpha}^{\text {short }}}{\partial R_{c}}\right\rangle+\sum_{\alpha}\left\langle\frac{\partial u_{\alpha}^{\text {es,nuc }}}{\partial R_{c}}\right\rangle \\
& +\sum_{p q} D_{p q} \sum_{i j} d_{i p} d_{j q} \frac{\partial}{\partial R_{c}}\{\mathrm{Eq} .(24)\} .
\end{aligned}
$$

The first two terms are given simply by averaging the gradient of the classical LJ and Coulomb potentials. In order to obtain the last term, it is necessary to evaluate the derivative of $\psi$ with respect to the nuclear coordinates. From Eq. (25), $\partial \psi / \partial x$ is interpolated by

$$
\frac{\partial \psi}{\partial x}=\frac{1}{\Delta} \sum_{l=1}^{3} \sum_{m=0}^{3} \sum_{n=0}^{3} A_{l m n}^{(i)} l x^{l-1} y^{m} z^{n},
$$

where $\Delta$ is the grid width. As a result, the interpolation of $\nabla \psi$ can be performed in the same way as for $\psi$.

For the compact basis functions, however, the free energy is expressed in terms of the multipole interactions. Thus, the gradients are evaluated by using the derivative of the multipoles and electrostatic fields.

\section{Computational details}

The present method was implemented in the computer code GAMESS. ${ }^{40}$ To investigate the solvation free energy and the solute electronic properties, we applied the present method to five molecules in aqueous solution: "solute" water, formaldehyde, acetone, benzonitrile, and nitrobenzene. In all the calculations, we employed the $(9 s 5 p 1 d / 4 s 1 p) /$ $[3 s 2 p 1 d / 2 s 1 p]$ basis set, ${ }^{41}$ which is of a valence double zeta plus polarization quality.
For water and formaldehyde, the calculations were performed with the conditions taken from Ref. 11 to compare the results with those by the MOZ-SCF, 1D-RISM-SCF, and PCM methods. The geometric parameters of the solute water molecule were $R_{\mathrm{OH}}=0.944 \AA$ and $\angle \mathrm{HOH}=106.7^{\circ}$. The geometry of formaldehyde was taken from Ref. 42: $R_{\mathrm{CO}}$ $=1.234 \AA, R_{\mathrm{CH}}=1.083 \AA$, and $\angle \mathrm{HCH}=118.0^{\circ}$. The SPClike $\operatorname{model}^{33}$ was employed to describe the solvent water molecule. The LJ parameters $\sigma=1.0 \AA$ and $\epsilon$ $=0.0545 \mathrm{kcal} / \mathrm{mol}$ were added to the water hydrogen sites in solute and solvent.

We employed the complete active space (CAS) SCF wave functions for acetone, benzonitrile, and nitrobenzene. For acetone, the active space was constructed by distributing six electrons in five active orbitals, the carbonyl $\pi, \pi^{*}, \sigma$, and $\sigma^{*}$, and the oxygen nonbonding $(n)$ orbitals, which are denoted by $(6,5)$. The active space for benzonitrile and nitrobenzene is $(10,10)$ and $(14,11)$, respectively. Note that the in-plane $\pi_{\mathrm{CN}}$ and $\pi_{\mathrm{CN}}^{*}$ orbitals in benzonitrile and the two oxygen lone pair orbitals in nitrobenzene are included in the active space in addition to the out-of-plane $\pi$ and $\pi^{*}$ orbitals. The solute $\mathrm{LJ}$ parameters for acetone and nitrobenzene were taken from the AMBER force field, ${ }^{43}$ while those of benzonitrile were taken from Ref. 44.

Vertical excitation energy calculations were performed for acetone, benzonitrile, and nitrobenzene molecules by the multireference Møller-Plesset (MRMP) perturbation method $^{45}$ to take the dynamic electron correlation effects into account. We used the solute Hamiltonian as

$$
\hat{H}=\hat{H}_{0}+\sum_{b} Z_{b} \psi\left(\mathbf{R}_{b}\right)-\psi(\mathbf{r}),
$$

where $H_{0}$ is the electronic Hamiltonian in the gas phase and $\psi(\mathbf{r})$ is the ESP coming from the solvent [Eq. (23)]. All $1 s$ orbitals of heavy atoms were kept frozen. The intruder state avoidance method ${ }^{46}$ was employed with the energy denominator shift parameter of 0.02 . A set of diffuse $p$ functions with the exponents of $0.034,0.048$, and 0.059 for $\mathrm{C}, \mathrm{N}$, and $\mathrm{O}$ atoms ${ }^{41}$ was added to each heavy atom in benzonitrile and nitrobenzene.

We applied the standard combination rule to construct the solute-solvent $\mathrm{LJ}$ potentials. The temperature was $298.15 \mathrm{~K}$ and the solvent density was set to be $0.03334 \AA^{-3}$. All the calculations were carried out on a $3.4 \mathrm{GHz}$ Pentium personal computer.

\section{RESULTS AND DISCUSSION}

\section{A. Newton-Raphson/Picard algorithm}

We examined the efficiency of the hybrid algorithm adopted here for solving the 3D-RISM equation. The Jacobian matrix was constructed at the initial step of iteration by using Eq. (A17) and it was used until the 3D-RISM-SCF is converged. The convergence was checked by the root mean square (rms) value of the residual, 


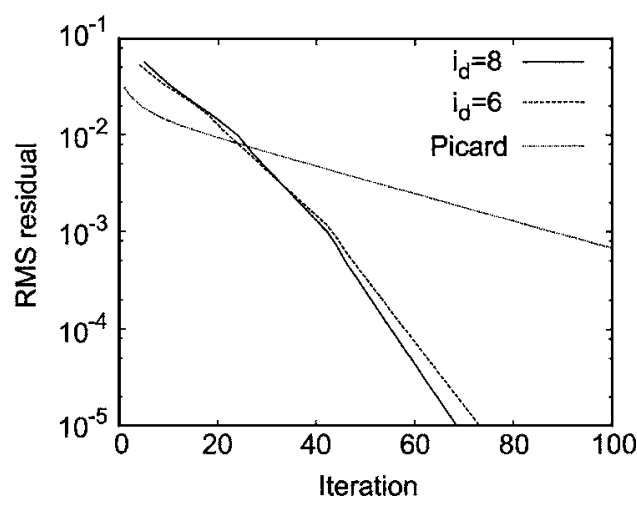

FIG. 1. Root mean square residual vs number of iterations for water in water. Solid and dashed lines are for the hybrid Newton-Rapshon/Picard algorithm with basis width $i_{d}=8$ and 6 , and the dotted one is for simple Picard algorithm.

$$
\left[\frac{1}{N_{v} N^{3}} \sum_{\alpha=1}^{N_{v}} \sum_{\mathbf{r}}^{N^{3}}\left|\theta_{\alpha}(\mathbf{r})-\theta_{\alpha}^{\prime}(\mathbf{r})\right|^{2}\right]^{1 / 2} .
$$

Here the results of a solute water are discussed. We employed $128^{3}$ cubic grid with the spacing of $0.159 \AA$. The core space determined based on the LJ parameters was composed of $49^{3}$ points. The most time-consuming step in calculating the Jacobian matrix is the multiplication and the backward FFT in the square bracket in Eq. (A17). The computational cost is proportional to $N_{v}^{2}$ times the number of basis functions $P_{s}(\mathbf{r})$. Thus, the calculations were carried out with two different basis functions. One is the basis width $i_{d}=8$, and another is $i_{d}=6$ defined in Eq. (A1). In the former case, the total number of basis functions was $5^{3}$, and the dimension of the Jacobian matrix $\operatorname{dim} J$ was 250 . In the latter case, the number of basis functions increased to $7^{3}$ and $\operatorname{dim} J=686$. The CPU time required for constructing the Jacobian matrix was 4.5 and $13 \mathrm{~min}$, respectively. Figure 1 shows the rms value of the residual versus the number of elementary iterations performed, where remarkable acceleration of the convergence is observed for the hybrid method compared with the simple PC iteration. The hybrid algorithm provides the rms residual less than $10^{-5}$ in 70 and 74 iterations for $i_{d}=8$ and 6, while the simple PC one requires at least 200 iterations. It is found that finer basis functions do not necessarily provide a faster convergence.

\section{B. Electrostatic interaction}

\section{Solute to solvent}

First, we discuss the timing results of the ESP calculations. Table I summarizes the CPU time for the construction of the ESP map. We denote the present method based on Eq. (21) and the multipole expansion as "Fourier transform $(\mathrm{FT})+$ multipole," and the exact evaluation of $A_{p q}(\mathbf{r})$ in Eq. (14) with the Rys quadrature ${ }^{47,48}$ as "Rys." For the Rys method, the computational cost depends on the number of grid points $\left(N^{3}\right)$ and basis functions $\left(N_{\text {bas }}\right)$, and thus scales as $N^{3} N_{\text {bas }}^{2}$, which is qualitatively reproduced in Table I. The FT part in the FT+multipole method is composed of the preparation of $\widetilde{n}(\mathbf{k})$ and the 3D-FFT in Eq. (21). We increased the box size for the FT part, as mentioned in Sec. II B. The number of grid points in the extended cell was set at 100 and 200 for $N=64$ and 128, respectively. As shown in Table I, the FT part takes more CPU time than the multipole expansion for smaller molecules, but the latter becomes dominant for larger ones. It accounts for $37 \%$ of the total CPU time for acetone, but $60 \%$ for nitrobenzene. The CPU time of the multipole part is in direct proportion to the number of multipole expansion. Since the maximum allowed exponent for the Fourier part is proportional to the inverse square of the grid width, the number of multipoles increases when a coarser grid is employed. In the case of acetone, for example, the number of multipole expansion increases by $16 \%$ when a coarser grid of spacing of $0.212 \AA$ is employed. The resultant CPU time increases at the same rate. For solute molecules considered here, a factor of 30-80 reduction in computational cost is achieved by the FT+multipole method compared to the Rys method. It is noteworthy that better performance is observed for larger molecules.

Next, we examine the accuracy of the FT+multipole method. Figure 2 shows the total potential in Eq. (12) divided by $k_{B} T$ for acetone and benzonitrile in aqueous solution. The potential calculated by the FT+multipole method reproduces that exactly evaluated by the Rys method very well. It is noted that the deep minima at the hydrogen site, which are important for a correct description of hydrogen bonding, are well reproduced. In the region near the solute, the accuracy of the FT+multipole method depends on the truncation of the multipole expansion. To check the multi-

TABLE I. CPU times (minutes) for electrostatic potential map construction per RISM-SCF cycle. FT + multipole refers to the present method based on Fourier transform and multipole expansion, and Rys to the Rys polynomial based method.

\begin{tabular}{|c|c|c|c|c|c|c|c|}
\hline \multirow[b]{2}{*}{ Molecule } & \multirow[b]{2}{*}{$N_{\text {bas }}$} & \multicolumn{2}{|c|}{ Grid $^{\mathrm{a}}$} & \multicolumn{3}{|c|}{ FT + multipole } & \multirow[b]{2}{*}{ Rys } \\
\hline & & $N$ & $\Delta$ & FT & Multipole $^{\mathrm{b}}$ & Total & \\
\hline Water & 25 & 128 & 0.159 & 0.1 & $0.1 \quad(26)$ & 0.2 & 5.3 \\
\hline \multirow[t]{3}{*}{ Acetone } & 90 & 64 & 0.318 & 0.0 & $0.1(405)$ & 0.1 & 7.6 \\
\hline & & 128 & 0.159 & 1.2 & $0.5(291)$ & 1.7 & 61.2 \\
\hline & & 128 & 0.212 & 1.0 & $0.6(338)$ & 1.6 & 61.2 \\
\hline Benzonitrile & 145 & 128 & 0.265 & 0.8 & $1.1(652)$ & 1.9 & 146.1 \\
\hline Nitrobenzene & 160 & 128 & 0.265 & 0.9 & $1.3(751)$ & 2.1 & 172.1 \\
\hline
\end{tabular}

Total number of grid is $N^{3}$ and $\Delta$ is grid width given in angstroms.

${ }^{b}$ Values in parentheses are the numbers of multipole expansion origins. 

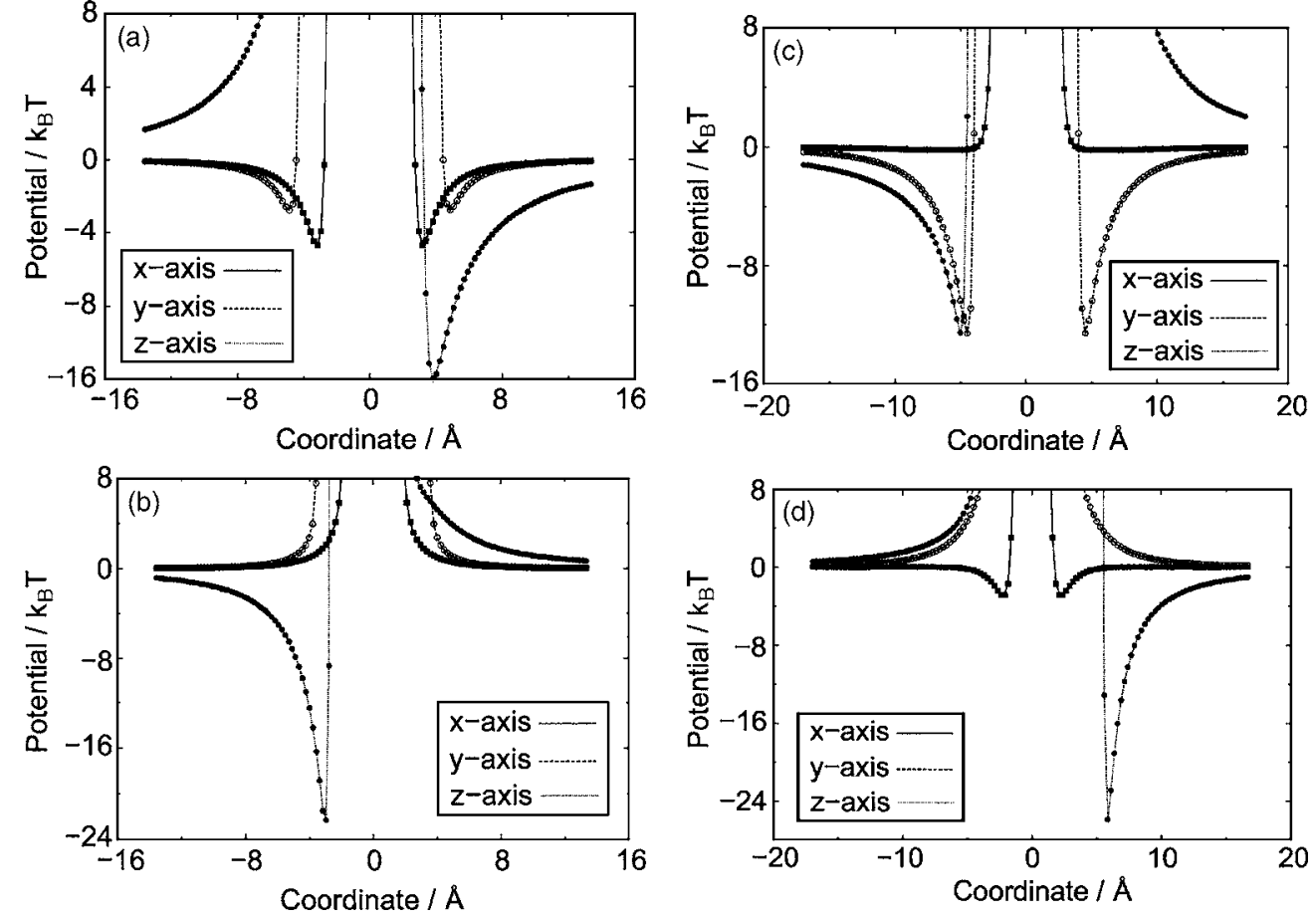

FIG. 2. Total interaction potential for acetone and benzonitrile in aqueous solution. (a) Acetone-oxygen site of water and (b) hydrogen site; (c) benzonitrileoxygen and (d) hydrogen sites. Lines refer to Fourier transform + multipole method and points to the Rys one. The solute molecule is placed in the $y z$ plane with molecular axis along the $z$ axis.

pole truncation error, we replaced the multipole part by the Rys quadrature, but little change was observed in the ESP, indicating that the truncation of the multipole expansion at the second order is a reasonable approximation. At the distant region, the ESP by the FT+multipole method approaches that by the Rys method, as shown in Fig. 2. Thus the correct asymptotic behavior of ESP is well reproduced.

\section{Solvent to solute}

We present timing data for estimating the matrix elements of the solute-solvent electrostatic interaction $h_{p q}^{\mathrm{es}}$. The CPU times for the $h_{p q}^{\text {es }}$ construction are given in Table II. We denote the present method due to Eq. (24) and the multipole interaction as "Gauss-Hermite $(\mathrm{GH})+$ multipole," and the exact evaluation of $A_{p q}(\mathbf{r})$ in Eq. (22) with the Rys quadrature as "Rys." As seen in Table II, the computational cost in the
Rys method is proportional to $N^{3} N_{\text {bas }}^{2}$ as in the case of the ESP calculation. For the $\mathrm{GH}+$ multipole method, the $\mathrm{GH}$ part is comparable to that of the multipole interaction for small molecules, and the latter becomes dominant for larger ones. For the solute molecules treated here, the present $\mathrm{GH}$ + multipole method is 3-30 times as fast as the Rys method, and it takes the CPU time comparable to the FT+multipole method employed for the solute to solvent route.

We calculated $h_{p q}^{\text {es }}$ by using two different methods employing the same CDF. In Figs. 3(a) and 3(b), we plot the correlation of $h_{p q}^{\text {es }}$ by the $\mathrm{GH}+$ multipole method versus that by the Rys method for acetone and nitrobenzene in aqueous solution, respectively. Good agreement between the matrix elements calculated by the two methods is clearly observed. The rms difference was $1.5 \times 10^{-5}$ and $1.2 \times 10^{-5}$ hartree for acetone and nitrobenzene. We obtained the solute energies by

TABLE II. CPU times (minutes) for solute-solvent interaction matrix construction per RISM-SCF cycle. GH + multipole refers to the present method based on the Gauss-Hermite quadrature and mutipole expansion, and Rys to the Rys polynomial based method.

\begin{tabular}{|c|c|c|c|c|c|c|c|}
\hline \multirow[b]{2}{*}{ Molecule } & \multirow[b]{2}{*}{$N_{\text {bas }}$} & \multicolumn{2}{|c|}{ Grid $^{\mathrm{a}}$} & \multicolumn{3}{|c|}{$\mathrm{GH}+$ multipole } & \multirow[b]{2}{*}{ Rys } \\
\hline & & $N$ & $\Delta$ & $\mathrm{GH}$ & Multipole $^{\mathrm{b}}$ & Total & \\
\hline Water & 25 & 128 & 0.159 & 0.7 & $0.1 \quad(26)$ & 0.7 & 2.4 \\
\hline \multirow[t]{3}{*}{ Acetone } & 90 & 64 & 0.318 & 0.1 & $0.1(405)$ & 0.2 & 3.4 \\
\hline & & 128 & 0.159 & 0.7 & $0.8(291)$ & 1.4 & 27.3 \\
\hline & & 128 & 0.212 & 0.6 & $0.9(338)$ & 1.5 & 27.4 \\
\hline Benzonitrile & 145 & 128 & 0.265 & 0.6 & $1.7(652)$ & 2.3 & 67.1 \\
\hline Nitrobenzene & 160 & 128 & 0.265 & 0.6 & $2.0(751)$ & 2.6 & 79.3 \\
\hline
\end{tabular}

${ }^{\mathrm{a}}$ Total number of grid is $N^{3}$ and $\Delta$ is grid width given in angstroms.

${ }^{b}$ Values in parentheses are the numbers of multipole expansion origins. 

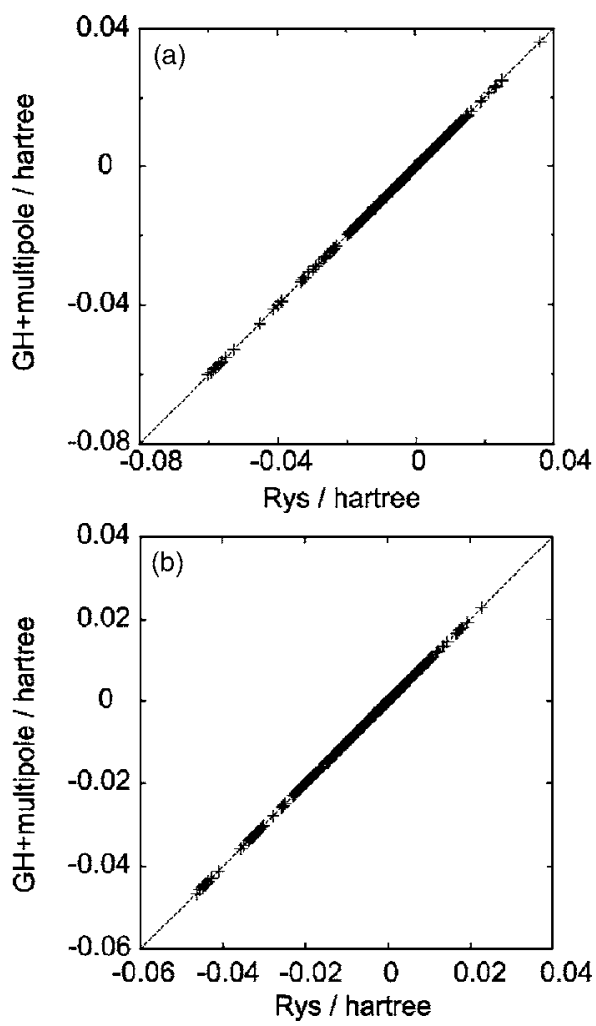

FIG. 3. Correlations of solute-solvent interaction matrix elements between the GH+multipole method and the Rys polynomial based method. (a) Acetone and (b) nitrobenzene in water.

the $\mathrm{GH}+$ multipole (Rys) method as follows; -192.90874 $(-192.90881)$ for acetone and $-435.82011(-435.81979)$ for nitrobenzene in water. The energy difference for all the molecules treated here is within $5 \times 10^{-4}$ hartree when the same CDF is employed.

\section{Energy gradient}

Table III presents timing results for the gradient calculation. We evaluated the gradient of $h_{p q}^{\text {es }}$ by using two different methods. For the Rys method, the CPU time is proportional to $N_{\text {bas }}^{2} N^{3}$, as in the cases of ESP. For the GH part in the $\mathrm{GH}+$ multipole method, it was found that the CPU time in the gradient computation is comparable to that in the interaction matrix calculation. Again, the estimation of the de- rivative of multipole interaction is a time-limiting step. It accounts for $80 \%$ of the total CPU time for benzonitrile and nitrobenzene. The computation of the gradient takes 1.1-1.4 times as much CPU time as that of the interaction matrix. In terms of the total CPU time, a factor of 20-160 reduction was obtained as compared to the Rys quadrature calculations.

\section{Comparison with other methods}

The present 3D-RISM-SCF method was applied to water and formaldehyde in aqueous solution. We employed $128^{3}$ cubic grid with the spacing of $0.159 \AA$, corresponding to the elementary cell of $20.3 \AA$. The calculations were carried out with the conditions taken from Ref. 11.

We compare the results with those from the MOZ-SCF, 1D-RISM-SCF, and PCM methods, which are summarized in Table IV. The solvation free energy is defined as the sum of the solute electronic reorganization energy and excess chemical potential $\Delta \mu$. The latter is expressed as

$$
\Delta \mu=-\frac{\rho}{\beta} \sum_{\alpha} \int d \mathbf{r}\left\{c_{\alpha}(\mathbf{r})-\frac{1}{2} h_{\alpha}^{2}(\mathbf{r})+\frac{1}{2} h_{\alpha}(\mathbf{r}) c_{\alpha}(\mathbf{r})\right\},
$$

and the average solute-solvent interaction energy $E_{\text {int }}$ is given by

$$
E_{\text {int }}=\sum_{\alpha} \int d \mathbf{r} \rho g_{\alpha}(\mathbf{r}) u_{\alpha}(\mathbf{r})
$$

It was found that the energy components calculated by the FT/GH+multipole method reproduce those by the Rys method within $0.1 \mathrm{kcal} / \mathrm{mol}$.

For water in the water system, the solvation free energy was calculated to be $-5.6 \mathrm{kcal} / \mathrm{mol}$, which is in agreement with the experimental estimate of $-6.3 \mathrm{kcal} / \mathrm{mol} .{ }^{49}$ The other methods gave the solvation free energies of $-5.1,-4.2$, and $-3.9 \mathrm{kcal} / \mathrm{mol}$ for the 1D-RISM-SCF, MOZ-SCF, and PCM, respectively. The absolute values of excess chemical potential and electronic reorganization energy are the largest for the 1D-RISM-SCF and the smallest for the PCM, indicating that the strength of the electric field acting on the solute increases in the order of $\mathrm{PCM}<\mathrm{MOZ}-\mathrm{SCF}$ $<3 \mathrm{D}-\mathrm{RISM}$-SCF $<1 \mathrm{D}$-RISM-SCF. This ordering was also found in the magnitude of the solute-solvent interaction. Furthermore, this tendency is reflected in the dipole moment

TABLE III. CPU times (minutes) for analytical free energy gradient. GH+multipole refers to the present method based on the Gauss-Hermite quadrature and multipole expansion, and Rys to the Rys polynomial based

\begin{tabular}{|c|c|c|c|c|c|c|c|}
\hline \multirow[b]{2}{*}{ Molecule } & \multirow[b]{2}{*}{$N_{\text {bas }}$} & \multicolumn{2}{|c|}{ Grid $^{\mathrm{a}}$} & \multicolumn{3}{|c|}{$\mathrm{GH}+$ multipole } & \multirow[b]{2}{*}{ Rys } \\
\hline & & $N$ & $\Delta$ & GH & Multipole $^{\mathrm{b}}$ & Total & \\
\hline Water & 25 & 128 & 0.159 & 0.7 & 0.1 (26) & 0.8 & 16.4 \\
\hline \multirow[t]{3}{*}{ Acetone } & 90 & 64 & 0.318 & 0.1 & $0.2(405)$ & 0.3 & 24.6 \\
\hline & & 128 & 0.159 & 0.7 & $1.2(291)$ & 1.8 & 196.4 \\
\hline & & 128 & 0.212 & 0.6 & $1.4(338)$ & 2.0 & 196.6 \\
\hline Benzonitrile & 145 & 128 & 0.265 & 0.6 & $2.6(652)$ & 3.3 & 491.2 \\
\hline Nitrobenzene & 160 & 128 & 0.265 & 0.6 & $3.0(751)$ & 3.7 & 583.1 \\
\hline
\end{tabular}
method.

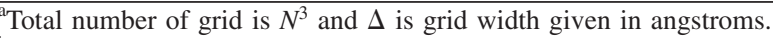

${ }^{b}$ Values in parentheses are the numbers of multipole expansion origins. 
TABLE IV. Solvation free energy and electronic properties of water and formaldehyde in aqueous solution. FT/GH refers to the present method based on the Fourier transform/Gauss-Hermite quadrature and multipole expansion, and Rys refers to the Rys polynomial based method.

\begin{tabular}{|c|c|c|c|c|c|}
\hline & \multicolumn{2}{|c|}{ 3D-RISM-SCF } & \multirow[b]{2}{*}{ 1D-RISM-SCF } & \multirow[b]{2}{*}{ MOZ-SCF ${ }^{\mathrm{a}}$} & \multirow[b]{2}{*}{$\mathrm{PCM}^{\mathrm{a}}$} \\
\hline & FT/GH & Rys & & & \\
\hline \multicolumn{6}{|c|}{ Water } \\
\hline Solvation free energy ${ }^{\mathrm{b}}$ & -5.6 & -5.6 & -5.1 & -4.2 & $-1.4(-3.9)$ \\
\hline Reorganization energy & 2.0 & 2.0 & 3.0 & 1.6 & $0.6(0.6)$ \\
\hline Excess chemical potential & -7.6 & -7.6 & -8.1 & -5.8 & $-2.0(-4.5)$ \\
\hline Solute-solvent interaction & -26.3 & -26.2 & -28.8 & -18.6 & $-14.0(-17.9)$ \\
\hline Dipole moment ${ }^{c}$ & 2.63 & 2.63 & 2.78 & 2.57 & $2.44(2.44)$ \\
\hline \multicolumn{6}{|c|}{ Formaldehyde } \\
\hline Solvation free energy & 1.9 & 1.9 & 4.1 & 1.3 & $2.7(-2.3)$ \\
\hline Reorganization energy & 1.9 & 1.8 & 2.5 & 0.9 & $0.2(0.3)$ \\
\hline Excess chemical potential & 0.0 & 0.1 & 1.6 & 0.4 & $2.5(-2.5)$ \\
\hline Solute-solvent interaction & -18.4 & -18.3 & -19.9 & -9.5 & $-8.7(-17.3)$ \\
\hline Dipole moment ${ }^{\mathrm{c}}$ & 3.27 & 3.27 & 3.42 & 3.03 & $2.75(2.74)$ \\
\hline
\end{tabular}

${ }^{\mathrm{a}}$ Reference 11. Values in parentheses are after the Pauli repulsion and dispersion correction.

${ }^{\mathrm{b}}$ Energies are given in $\mathrm{kcal} / \mathrm{mol}$.

${ }^{\mathrm{c}}$ Given in debye.

enhancement. Compared with the gas phase value of $2.19 \mathrm{D}$, the dipole moment increased by $0.44,0.59,0.38$, and $0.25 \mathrm{D}$ for the 3D-RISM-SCF, 1D-RISM-SCF, MOZ-SCF, and PCM, respectively. It is noted that Krienke et al. developed new water potential parameters which reproduce the experimental thermodynamic properties of the same system within the framework of the 1D-RISM-SCF method. ${ }^{50}$

For formaldehyde in aqueous solution, the solvation free energy was estimated to be $1.9 \mathrm{kcal} / \mathrm{mol}$ by the 3D-RISMSCF method though the experimental value was negative, $-1.7 \mathrm{kcal} / \mathrm{mol}^{51}$ The corresponding values for $1 \mathrm{D}-\mathrm{RISM}$ SCF, MOZ-SCF, and PCM were 4.1, 1.3, and $2.7 \mathrm{kcal} / \mathrm{mol}$, respectively. The solute-solvent interaction energy showed a similar trend as in the case of water solute. Compared with the 3D-RISM-SCF method, the 1D-RISM-SCF gave a slightly larger value, while it was considerably smaller for the MOZ-SCF and PCM. Furthermore, this trend is clearly seen in the solute electronic polarization due to the solvation. The degrees of dipole moment enhancement were $0.79,0.92$, 0.55, and 0.27 D for 3D-RISM-SCF, 1D-RISM-SCF, MOZSCF, and PCM, respectively.

\section{Solvatochromic shifts}

\section{Solvent distributions}

We performed the 3D-RISM-CASSCF calculations for acetone, benzonitrile, and nitrobenzene in aqueous solution. Here we compared the 3D-RISM-SCF results with those of the 1D-RISM-SCF method. For all the solute molecules considered here, the geometrical parameters optimized by the two different 3D-RISM-SCF methods agree within $0.001 \AA$, and they are also close to those by the 1D-RISM-SCF method. The energy components obtained by the FT/GH + multipole method are in very good agreement with those by the Rys method, and their difference is within $0.1 \mathrm{kcal} / \mathrm{mol}$.

Figures 4-6 show the water oxygen and hydrogen distributions obtained for the optimized geometries of acetone, benzonitrile, and nitrobenzene in solution, respectively. All the molecules were placed in the $y z$ plane with molecular axis along the $z$ axis. For the acetone-water profile, the water oxygen site is attracted to the positively charged carbonyl and methyl carbon atoms. As shown in Figs. 4(a) and 4(c), the oxygen site approaches perpendicularly the carbonyl carbon. In addition, the water oxygen peak is located between the methyl groups. The water hydrogen site has a large density around the carbonyl oxygen. As seen in Figs. 4(b) and 4(d), it interacts strongly with the oxygen $n$ orbital rather than with the carbonyl $\pi$ orbital. Comparing the positions of intense hydrogen peaks with those of oxygen peaks in Fig. 4(a), we can find that the hydrogen bond is formed by donating one of the $\mathrm{OH}$ bonds of water molecule to the carbonyl oxygen.

In the benzonitrile-water profile, the water oxygen site is attracted to the positively charged hydrogen and cyano carbon atoms, and the corresponding six peaks appear between these atoms in Figs. 5(a) and 5(c). In addition, the oxygen peaks are located above the benzene plane rather than the cyano carbon in spite of its large positive charge. Since these peaks were still observed when the electrostatic interaction was turned off in solving the 3D-RISM equations, they are attributed to the packing effect. As seen in Figs. 5(b) and $5(\mathrm{~d})$, the water hydrogen interacts strongly with the nitrogen $n$ orbital by forming the hydrogen bond, while such a strong peak was not observed for the cyano $\pi$ orbitals.

For the nitrobenzene-water profile, the water oxygen site distribution is similar to that of benzonitrile. As shown in Figs. 6(a) and 6(c), we observe six peaks between the positively charged hydrogen and nitrogen atoms, and two peaks above the benzene ring: The water hydrogen site interacts strongly with the oxygen $n$ orbitals by hydrogen bonding, as seen in Figs. 6(b) and 6(d). 

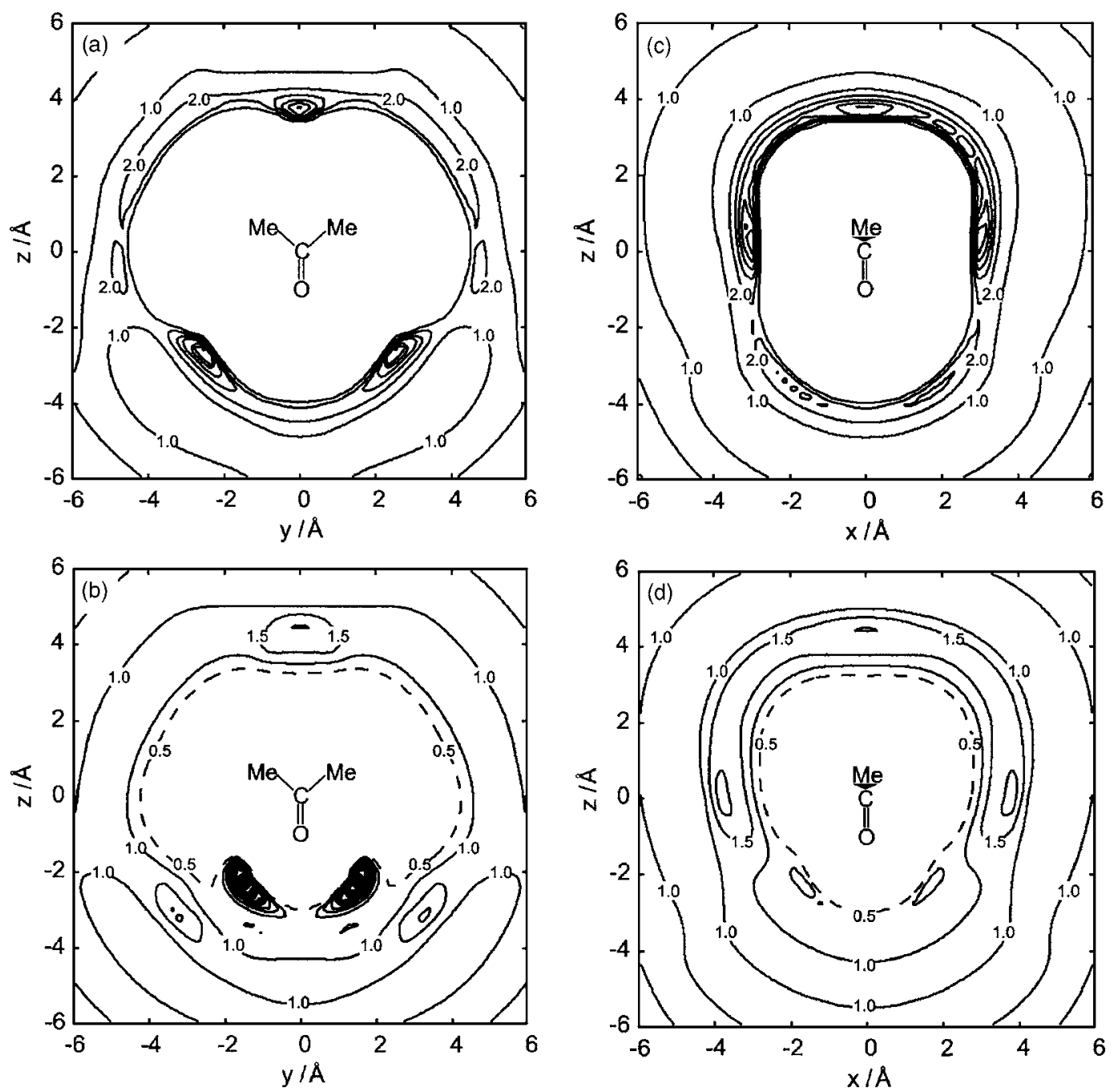

FIG. 4. Solvent distribution function around solute acetone. (a) and (c) for oxygen site and (b) and (d) for hydrogen. Oxygen atom is located at (0,0, $-1.269)$, carbonyl carbon at $(0,0,-0.030)$, and methyl carbon atoms at $(0, \pm 1.291,0.741)$. Coordinates are given in angstroms.

\section{Solvation free energies}

It is well known that the RISM theory with the HNC closure overestimates the cavity formation energy. Actually, the 1D-RISM-SCF calculations gave positive solvation free energies of $14.9,22.8$, and $23.8 \mathrm{kcal} / \mathrm{mol}$ for acetone, benzonitrile, and nitrobenzene, respectively, while the corresponding experimental values ${ }^{51-53}$ are $-3.8,-4.1$, and $-4.2 \mathrm{kcal} / \mathrm{mol}$. With the 3D-RISM-SCF method, the degree of the overestimation was largely reduced and the resultant solvation free energies became 5.2, 10.5, and $10.3 \mathrm{kcal} / \mathrm{mol}$ for those solute molecules. In spite of the improvements, the 3D-RISM-SCF still overestimates the cavity formation energy. The solute-solvent interaction energies were calculated to be $-26.8,-30.1$, and $-29.0 \mathrm{kcal} / \mathrm{mol}$ for acetone, benzonitrile, and nitrobenzene, respectively, by the 3D-RISMSCF, which were comparable to the 1D-RISM-SCF results, $-29.6,-34.3$, and $-30.0 \mathrm{kcal} / \mathrm{mol}$. The dipole moment in aqueous solution was calculated as follows: $4.37,5.72$, and 5.31 D for the 1D-RISM-SCF and 4.13, 5.67, and 5.48 D for the 3D-RISM-SCF, indicating that the 3D-RISM-SCF method gave slightly smaller solute electronic polarization effect than the 1D-RISM-SCF.

\section{Vertical transition energies}

The calculated vertical excitation energies are summarized in Table $\mathrm{V}$ along with the experimental data. For acetone, we obtained the $n \pi^{*}$ transition energy of $4.41 \mathrm{eV}$ in the gas phase, which is comparable to the experimental estimate of $4.3-4.5 \mathrm{eV}$. In aqueous solution, it increased by $0.38(0.38)$ and $0.31 \mathrm{eV}$ for the 3D-RISM FT/GH (Rys) and 1D-RISM methods, corresponding to the blueshifts of 3030 (3060) and $2480 \mathrm{~cm}^{-1}$, which are overestimated in comparison with the experimental value. The blueshift for the $n \pi^{*}$ transition is attributed to the decrease in the dipole moment caused by the electronic excitation. The dipole moment became small by 1.7 and $1.9 \mathrm{D}$ for the 3D and 1D-RISM methods. It is noteworthy that a larger decrease in the dipole moment does not always cause a larger blueshift. The 3DRISM-SCF blueshift reflects a specific stabilization of the oxygen $n$ orbital as observed in the solvent distribution.

For benzonitrile, the following absorption bands were observed in the gas phase experimentally: ${ }^{54,55} 4.53(\epsilon$ $\simeq 800$ ), $\sim 5.6$ (intense, $\epsilon \simeq 2.5 \times 10^{4}$ ), and $\sim 6.6 \mathrm{eV}$ (broad, $\left.\epsilon \simeq 6 \times 10^{4}\right)$. Although the semiempirical calculations ${ }^{56-58}$ have assigned these bands to the transitions to the $1{ }^{1} B_{2}$, 

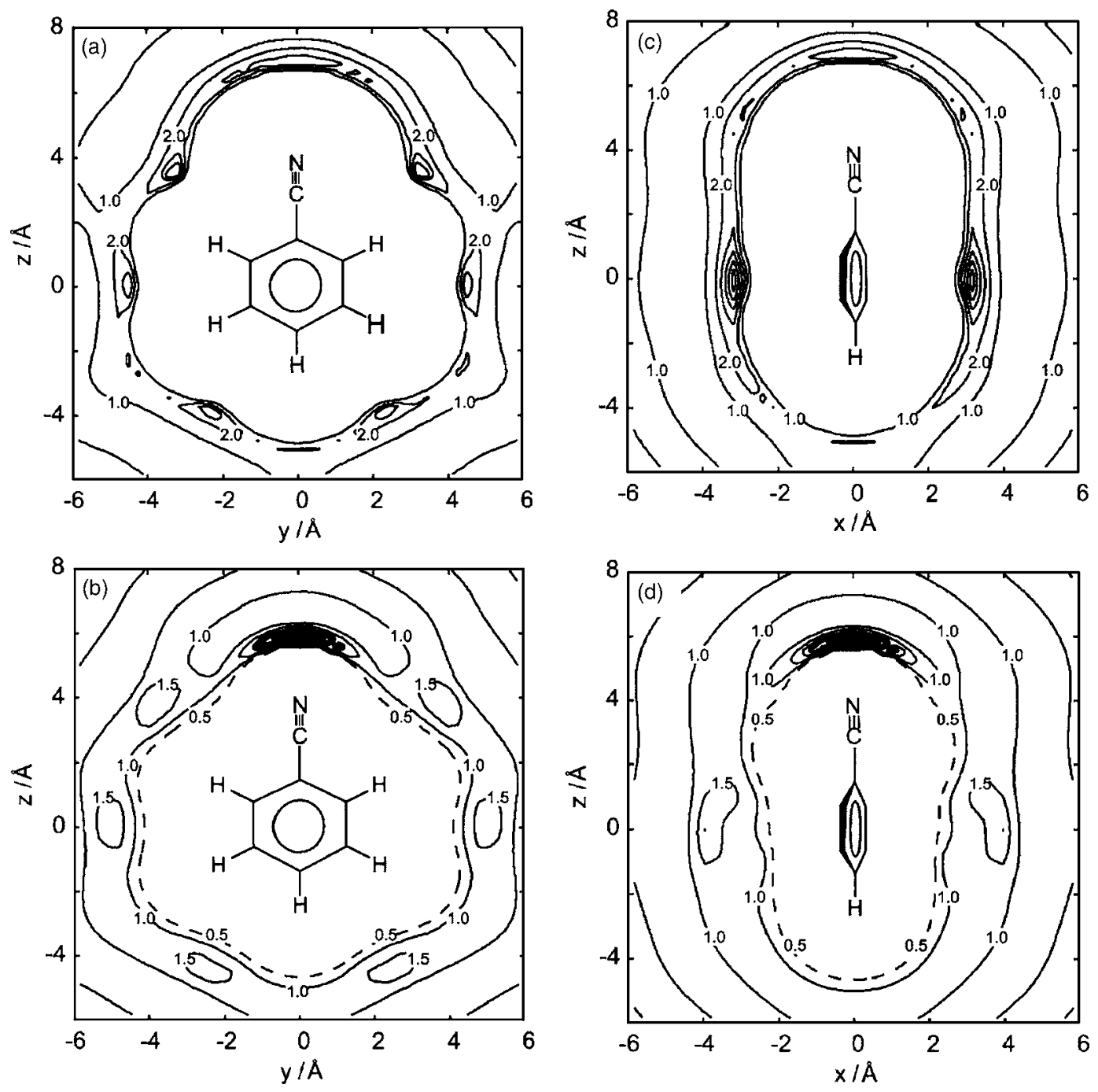

FIG. 5. Solvent distribution function around solute benzonitrile. For (a)-(d), see Fig. 4. Nitrogen atom is located at $(0,0,3.995)$, cyano carbon at $(0,0,2.833)$, $\mathrm{C}_{1}$ (adjacent to cyano group) at $(0,0,1.386), \mathrm{C}_{2}$ and $\mathrm{C}_{6}$ at $(0, \pm 1.219,0.696), \mathrm{C}_{3}$ and $\mathrm{C}_{5}$ at $(0, \pm 1.213,-0.700)$, and $\mathrm{C}_{4}$ at $(0,0,-1.397)$.

$2{ }^{1} A_{1}$, and $2{ }^{1} B_{2}$ excited states, the recent CASPT2 calculations $^{59}$ gave only the two transitions in this energy range, the vertical transition energies of 4.64 and $6.24 \mathrm{eV}$ to the $1{ }^{1} B_{2}$ and $2{ }^{1} A_{1}$ states, respectively.

In the $C_{2 v}$ point group, the ground state is mainly described by the electronic configuration $\cdots\left(1 b_{1}\right)^{2}\left(2 b_{1}\right)^{2}\left(8 b_{2}\right)^{2}\left(1 a_{2}\right)^{2}\left(3 b_{1}\right)^{2}$ and the $1^{2} B_{2}$ state is described by the singly excited configurations from $1 a_{2}$ to $4 b_{1}$ and from $3 b_{1}$ to $2 a_{2}$. As seen in Table V, the present MRMP calculations provided the excitation energy of $4.52 \mathrm{eV}$ in the gas phase, which is in good agreement with the experiment. Since the dipole moment of this state is very close to that of the ground state, no significant solvatochromic shift was observed in the present calculations.

The $2{ }^{1} A_{1}$ state is mainly described by the singly excited configuration from $3 b_{1}$ to $4 b_{1}$ and has a strong chargetransfer character. The gas phase excitation energy to this state was calculated to be $6.34 \mathrm{eV}$, which is close to the CASPT2 result. In aqueous solution, the vertical transition energy was decreased by $0.27(0.21)$ and $0.25 \mathrm{eV}$ for the 3D-FT/GH (Rys) and 1D-RISM methods, respectively, corresponding to the redshifts of 2220 (1740) and $1990 \mathrm{~cm}^{-1}$.
Although a small difference is observed between the two different 3D-RISM-SCF methods, this came from the dynamic electron correlation energy. At the 3D-RISMCASSCF level, the difference in redshift was $\sim 100 \mathrm{~cm}^{-1}$.

Finally, we discuss the excitation energies of nitrobenzene. Since the absorption spectrum is generally very broad and featureless in any solvent and even in vacuum, details of the excited states have not been clarified. Therefore, it is important to obtain reliable knowledge of the energetic positions of the electronically excited states by accurate $a b$ initio electronic structure method. The recent CASPT2 calculations provided the following transition energies: $:^{60} 3.57\left(1{ }^{1} A_{2}\right)$, $4.14\left(1{ }^{1} B_{1}\right), 4.40\left(1{ }^{1} B_{2}\right), 4.99\left(2{ }^{1} A_{1}\right)$, and $5.59 \mathrm{eV}\left(2{ }^{1} B_{2}\right)$.

The ground state electronic configuration is $\cdots\left(1 b_{1}\right)^{2}\left(2 b_{1}\right)^{2}\left(16 a_{1}\right)^{2}\left(11 b_{2}\right)^{2}\left(1 a_{2}\right)^{2}\left(3 b_{1}\right)^{2}\left(2 a_{2}\right)^{2}$. The vertical $S_{1}$ state is of pure $n \pi^{*}$ character, which is mainly described by the singly excited configuration from $11 b_{2}$ to $4 b_{1}$ orbitals localized at the nitro group. The character of the $S_{1}$ state has been a long-standing question experimentally and theoretically. ${ }^{55,61,62}$ The vertical excitation energy in vacuum was calculated to be $3.58 \mathrm{eV}$, which is in good agreement with the experimental value in hexane. The direction of sol- 

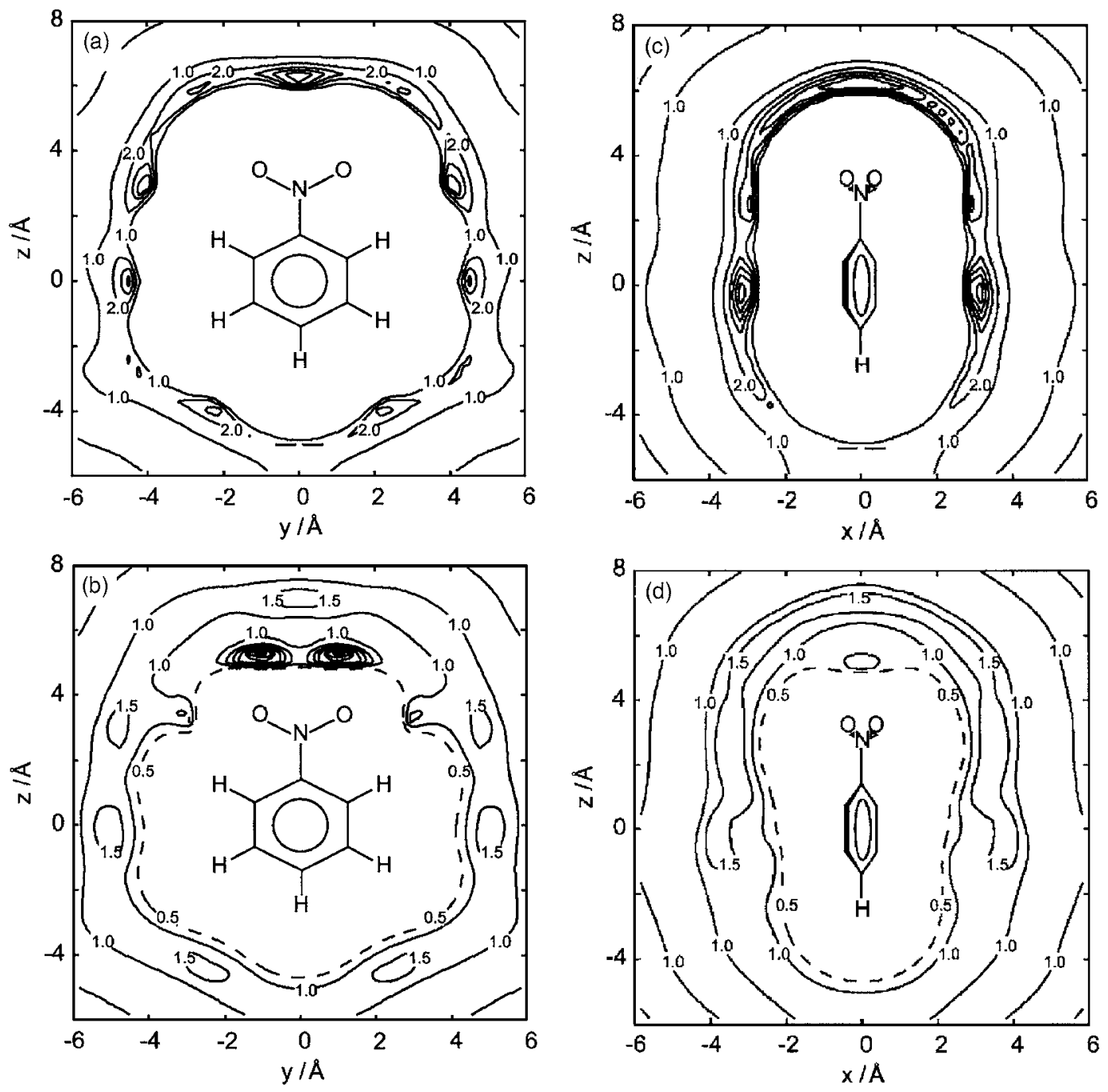

FIG. 6. Solvent distribution function around solute nitrobenzene. For (a)-(d), see Fig. 4. Nitrogen atom is located at $(0,0,2.785)$, oxygen atoms at $(0, \pm 1.063,3.362), \mathrm{C}_{1}$ (adjacent to nitro group) at $(0,0,1.326), \mathrm{C}_{2}$ and $\mathrm{C}_{6}$ at $(0, \pm 1.222,0.656), \mathrm{C}_{3}$ and $\mathrm{C}_{5}$ at $(0, \pm 1.213,-0.740)$, and $\mathrm{C}_{4}$ at $(0,0,-1.438)$.

vatochromic shift of this band in polar solvents has been also a matter of discussion. ${ }^{62}$ As seen in Table V, the 3D-RISMSCF calculations gave a very small blueshift of $0.07 \mathrm{eV}$ in spite of a rather large decrease of the dipole moment upon excitation, $1.57 \mathrm{D}$. It is noted that the 1D-RISM-SCF gave a similar value of the blueshift.

The second excited state is attributed to the excitations from $16 a_{1}$ to $4 b_{1}$ and from $1 a_{2}$ and $11 b_{2}$ to $4 b_{1}$ orbitals. The excitation is also localized mainly at the nitro group. The vertical transition energies was increased by $0.16 \mathrm{eV}$ due to the solvation. This corresponds to the blueshift of $\sim 1300 \mathrm{~cm}^{-1}$. The $S_{3}$ state is of a $\pi \pi^{*}$ character and is mainly described by the singly excited configurations of $3 b_{1} \rightarrow 3 a_{2}$ and $2 a_{2} \rightarrow 5 b_{1}$. Since the dipole moment of this state is close to that of the ground state, the solvatochromic shift is very small.

The $S_{4}$ state is a strong charge-transfer state. In the gas phase, the excitation energy was estimated to be $5.50 \mathrm{eV}$, which overestimates the experimental value by $0.33 \mathrm{eV}$. As expected from the large increase in dipole moment due to the excitation, the 3D FT/GH (Rys) and 1D-RISM methods provided the redshifts of 4170 (4110) and $3720 \mathrm{~cm}^{-1}$, respec- tively. It is noted that the calculated redshift agrees very well with the experimental value, $\sim 4000 \mathrm{~cm}^{-1}$, in spite of the overestimation of the excitation energy itself.

The magnitude of shifts in aprotic solvents is sometimes related to the dipole moments of the ground and excited states, $\boldsymbol{\mu}_{0}$ and $\boldsymbol{\mu}_{i}$, as $\Delta E_{i} \propto \boldsymbol{\mu}_{0} \cdot\left(\boldsymbol{\mu}_{0}-\boldsymbol{\mu}_{i}\right)$. The calculated shifts for nitrobenzene in Table V significantly deviate from such a relation, indicating the importance of hydrogen bonding in determining the solvatochromic shifts.

\section{CONCLUSION}

In the present paper, we presented an efficient implementation of the 3D-RISM-SCF method. A robust and efficient algorithm was developed for solving the 3D-RISM equation. The algorithm is a hybrid NR/PC method with the analytical Jacobian matrix. A remarkable acceleration in the convergence was observed in comparison with the simple PC iterations.

We discussed efficient calculations of the solute-solvent electrostatic interaction. For the solute to solvent route, the ESP map on a 3D grid was constructed directly by the electron density in reciprocal space and the multipole expansion. 
TABLE V. Vertical transition energies and dipole moments (values in parentheses) of acetone, benzonitrile, and nitrobenzene both in gas and aqueous solution phases. Units: $\mathrm{eV}$ for transition energies; debye for dipole moments.

\begin{tabular}{|c|c|c|c|c|c|c|}
\hline \multirow[b]{2}{*}{ State } & \multicolumn{2}{|l|}{ Gas } & \multicolumn{4}{|c|}{ Aqueous solution } \\
\hline & Calc. & Expt. & 3D FT/GH & 3D Rys & 1D-RISM & Expt. \\
\hline \multicolumn{7}{|c|}{ Acetone } \\
\hline $1^{1} A_{1}$ & $(2.86)$ & & (4.13) & $(4.12)$ & $(4.37)$ & \\
\hline $1{ }^{1} A_{2}$ & $4.41(1.39)$ & $4.49^{\mathrm{a}}$ & $4.78(2.41)$ & $4.79(2.40)$ & $4.72(2.50)$ & $4.68^{\mathrm{a}}$ \\
\hline \multicolumn{7}{|c|}{ Benzonitrile } \\
\hline $1{ }^{1} A_{1}$ & $(4.37)$ & & (5.75) & $(5.75)$ & $(5.82)$ & \\
\hline${ }^{1}{ }^{1} B_{2}$ & $4.52(4.31)$ & $4.53^{\mathrm{b}}$ & $4.53(5.69)$ & $4.54(5.69)$ & $4.54(5.78)$ & \\
\hline $2{ }^{1} A_{1}$ & $6.34(7.27)$ & & $6.07(9.46)$ & $6.13(9.41)$ & $6.09(9.40)$ & \\
\hline \multicolumn{7}{|c|}{ Nitrobenzene } \\
\hline $1{ }^{1} A_{1}$ & $(4.36)$ & & $(5.61)$ & $(5.60)$ & $(5.44)$ & \\
\hline $1{ }^{1} A_{2}$ & $3.58(2.75)$ & $3.65^{\mathrm{c}}$ & $3.65(4.04)$ & $3.65(4.04)$ & $3.63(3.93)$ & \\
\hline${ }^{1} B_{1}$ & $4.25(2.50)$ & & $4.41(3.78)$ & $4.41(3.77)$ & $4.41(3.77)$ & \\
\hline $1{ }^{1} B_{2}$ & $4.50(4.65)$ & $4.38^{\mathrm{d}}$ & $4.40(6.17)$ & $4.40(6.16)$ & $4.44(5.95)$ & \\
\hline $2{ }^{1} A_{1}$ & $5.50(12.48)$ & $5.17^{\mathrm{d}}$ & 4.98 (13.91) & $4.99(13.91)$ & $5.03(13.84)$ & $4.64^{\mathrm{d}}$ \\
\hline $2{ }^{1} B_{2}$ & $6.13(1.24)$ & & $6.23(3.06)$ & $6.23(3.06)$ & $6.22(2.80)$ & \\
\hline
\end{tabular}

It was confirmed that the resultant ESP map reproduces fairly well the exact one estimated by the quadratures with Rys polynomials. The present FT+multipole method was 30-80 times as fast as the Rys method. For the solvent to solute route, matrix elements of the solute-solvent interaction were evaluated directly by the numerical integration with the $\mathrm{GH}$ quadrature. The resultant matrix was in good agreement with one exactly evaluated by the Rys quadrature. We obtained a factor of 3-30 reduction in the computational cost by the present $\mathrm{GH}+$ multipole method. Furthermore, the first derivative of free energy with respect to the solute nuclear coordinates was implemented. A factor of 20-160 reduction in computational cost was obtained by the $\mathrm{GH}+$ multipole method. It is noteworthy that the present method works better as the molecular size increases.

We applied the present method to five solute molecules in aqueous solution; solute water, formaldehyde, acetone, benzonitrile, and nitrobenzene in aqueous solution. The results obtained by the two different 3D-RISM-SCF methods agreed very well. Compared with the MOZ-SCF, 1D-RISMSCF, and PCM methods, the 3D-RISM-SCF method exerts moderate electronic polarization effects on the solute electronic structure and the strength of the solvent electrostatic field increases in the order of $\mathrm{PCM}<\mathrm{MOZ}-\mathrm{SCF}$ $<3$ D-RISM-SCF $<1$ D-RISM-SCF. We also calculated the solvatochromic shifts and compared them with the experimental and other theoretical results.

As expected from the remarkable reduction in the computational cost observed here, the present implementation is highly suitable to large molecules for which the application of the 3D-RISM-SCF method with the direct calculations of solute-solvent electrostatic interactions is too time consum- ing. It provides efficient and useful tools for studying the electronic and thermodynamic properties of such molecules in solution.

\section{ACKNOWLEDGMENT}

This work was supported by the Grant-in-Aid from the Ministry of Education, Culture, Sports, Science and Technology in Japan.

\section{APPENDIX: JACOBIAN MATRIX}

The analytical expression of the Jacobian matrix can be easily derived as in the case of the site-site RISM equation. ${ }^{29,30,32}$ In the following, the arguments $\mathbf{r}$ and $\mathbf{k}$ refer to the discretized mesh point in real and reciprocal spaces, respectively. First, we introduce basis functions $P_{s}(\mathbf{r})$, which are defined as the 3D generalized "roof functions,"

$$
P_{s}(\mathbf{r})= \begin{cases}1 & \text { for } \mathbf{r} \in I_{0}^{s}=\left\{\mathbf{R}_{s}\right\} \\ \left(i_{d}-i\right) / i_{d} & \text { for } \mathbf{r} \in I_{i}^{s}-I_{i-1}^{s}\left(1 \leqslant i \leqslant i_{d}\right) \\ 0 & \text { otherwise, }\end{cases}
$$

where $\mathbf{R}_{s}$ is the center of the $s$ th basis and $I_{i}^{S}=\left\{\mathbf{r} ;\left|x-X_{s}\right|\right.$ $\left.\leqslant i \Delta,\left|y-Y_{s}\right| \leqslant i \Delta,\left|z-Z_{s}\right| \leqslant i \Delta\right\}$. Then the conjugate basis functions $Q_{s}(\mathbf{r})$ are introduced, which are linear combination of $P_{s}(\mathbf{r})$,

$$
Q_{s}=\sum_{t} B_{s t} P_{t}(\mathbf{r})
$$

such that 


$$
\sum_{\mathbf{r}} Q_{s}(\mathbf{r}) P_{t}(\mathbf{r})=\delta_{s t}
$$

The matrix $B$ is given by

$$
\left(B^{-1}\right)_{s t}=\sum_{\mathbf{r}} P_{s}(\mathbf{r}) P_{t}(\mathbf{r}) .
$$

From Eqs. (10) and (A2), it is easy to show that the coefficients $a_{\alpha s}$ are obtained by

$$
a_{\alpha s}=\sum_{\mathbf{r}} Q_{s}(\mathbf{r}) \theta_{\alpha}(\mathbf{r})
$$

The Jacobian matrix is defined by

$$
J_{\alpha s, \mu t}=\frac{\partial\left(a_{\alpha s}-a_{\alpha s}^{\prime}\right)}{\partial a_{\mu t}}=\delta_{\alpha \mu} \delta_{s t}-\frac{\partial a_{\alpha s}^{\prime}}{\partial a_{\mu t}},
$$

and the second term is

$$
\frac{\partial a_{\alpha s}^{\prime}}{\partial a_{\mu t}}=\sum_{\mathbf{r}} \sum_{\mathbf{r}^{\prime}} Q_{s}(\mathbf{r}) \frac{\partial \theta_{\alpha}^{\prime}(\mathbf{r})}{\partial \theta_{\mu}\left(\mathbf{r}^{\prime}\right)} P_{t}\left(\mathbf{r}^{\prime}\right) .
$$

The partial derivative $\partial \theta_{\alpha}^{\prime} / \partial \theta_{\mu}$ is obtained by employing the chain rule,

$$
\frac{\partial \theta_{\alpha}^{\prime}(\mathbf{r})}{\partial \theta_{\mu}\left(\mathbf{r}^{\prime}\right)}=\sum_{\mathbf{k}} \frac{\partial \theta_{\alpha}^{\prime}(\mathbf{r})}{\partial \tilde{\theta}_{\alpha}(\mathbf{k})} \frac{\partial \widetilde{\theta}_{\alpha}(\mathbf{k})}{\partial \widetilde{c}_{\mu}^{s}(\mathbf{k})} \frac{\partial \widetilde{c}_{\mu}^{s}(\mathbf{k})}{\partial c_{\mu}^{s}\left(\mathbf{r}^{\prime}\right)} \frac{\partial c_{\mu}^{s}\left(\mathbf{r}^{\prime}\right)}{\partial \theta_{\mu}\left(\mathbf{r}^{\prime}\right)} .
$$

The partial derivative terms are determined by the 3D-RISM equation in Eq. (6), the 3D-HNC closure in Eq. (7), and the discrete 3D Fourier transformations,

$$
\begin{aligned}
& \frac{\partial \widetilde{\theta}_{\alpha}(\mathbf{k})}{\partial \widetilde{c}_{\mu}^{s}(\mathbf{k})}=\widetilde{H}_{\mu \alpha}^{V V}(k)-\delta_{\alpha \mu} \\
& \frac{\partial c_{\mu}^{s}\left(\mathbf{r}^{\prime}\right)}{\partial \theta_{\mu}\left(\mathbf{r}^{\prime}\right)}=\exp \left[\gamma_{\mu}\left(\mathbf{r}^{\prime}\right)+\phi_{\mu}^{*}\left(\mathbf{r}^{\prime}\right)+\theta_{\mu}\left(\mathbf{r}^{\prime}\right)\right]-1 \\
& \frac{\partial \theta_{\alpha}^{\prime}(\mathbf{r})}{\partial \widetilde{\theta}_{\alpha}(\mathbf{k})}=\frac{1}{V} \exp (-i \mathbf{k} \cdot \mathbf{r}) \\
& \frac{\partial \widetilde{c}_{\mu}^{s}(\mathbf{k})}{\partial c_{\mu}^{s}\left(\mathbf{r}^{\prime}\right)}=\Delta^{3} \exp \left(i \mathbf{k} \cdot \mathbf{r}^{\prime}\right)
\end{aligned}
$$

Combining the above four derivative terms, we obtain

$$
\frac{\partial \theta_{\alpha}^{\prime}(\mathbf{r})}{\partial \theta_{\mu}\left(\mathbf{r}^{\prime}\right)}=\Delta^{3} D_{\mu \alpha}\left(\mathbf{r}-\mathbf{r}^{\prime}\right) \frac{\partial c_{\mu}^{s}\left(\mathbf{r}^{\prime}\right)}{\partial \theta_{\mu}\left(\mathbf{r}^{\prime}\right)},
$$

where

$$
D_{\mu \alpha}(\mathbf{r})=\frac{1}{V} \sum_{\mathbf{k}}\left[\widetilde{H}_{\mu \alpha}^{V V}(k)-\delta_{\alpha \mu}\right] e^{-i \mathbf{k} \cdot \mathbf{r}}
$$

Inserting Eq. (A10) to Eq. (A7), and using Eq. (A6), the Jacobian matrix is given by

$$
J_{\alpha s, \mu t}=\delta_{\alpha \mu} \delta_{s t}-\Delta^{3} \sum_{\mathbf{r}} \sum_{\mathbf{r}^{\prime}} Q_{s}(\mathbf{r}) D_{\mu \alpha}\left(\mathbf{r}-\mathbf{r}^{\prime}\right) \frac{\partial c_{\mu}^{s}\left(\mathbf{r}^{\prime}\right)}{\partial \theta_{\mu}\left(\mathbf{r}^{\prime}\right)} P_{t}\left(\mathbf{r}^{\prime}\right) .
$$

Note that in the present formulation the Jacobian matrix depends on $\theta_{\mu}(\mathbf{r})$ and must be calculated repeatedly. This is because we define the larger core space as the coarse part and the assumption of $\partial c_{\mu}^{s} / \partial \theta_{\mu} \simeq-1$ is not always valid. In the actual computation, however, we construct the Jacobian matrix only once at the beginning of the 3D-RISM-SCF cycle. The same matrix is employed for solving the 3DRISM equations until the 3D-RISM-SCF is converged. This is partly because much computational cost is required. Although $D_{\mu \alpha}$ are easily constructed by performing the backward 3D-FFT of $\tilde{H}_{\mu \alpha}^{V V}-\delta_{\alpha \mu}$, the time-limiting step is the double summation over $\mathbf{r}$ and $\mathbf{r}^{\prime}$. Therefore, we convert the second term in the right-hand side of Eq. (A12) to a more useful form. Using Eq. (A2), we can rewrite the second term of the Jacobian matrix in Eq. (A12) without $Q_{s}$,

$$
-\sum_{w} B_{s w}\left[\Delta^{3} \sum_{\mathbf{r}} \sum_{\mathbf{r}^{\prime}} P_{w}(\mathbf{r}) D_{\mu \alpha}\left(\mathbf{r}-\mathbf{r}^{\prime}\right) \frac{\partial c_{\mu}^{s}\left(\mathbf{r}^{\prime}\right)}{\partial \theta_{\mu}\left(\mathbf{r}^{\prime}\right)} P_{t}\left(\mathbf{r}^{\prime}\right)\right] .
$$

Even in this form the summation over $\mathbf{r}$ is reduced drastically because $P_{w}(\mathbf{r})$ have nonzero values at $\left(2 i_{d}-1\right)^{3}$ points while $Q_{s}(\mathbf{r})$ are determined in the whole core space. Further reduction in the computational cost is possible. The double summation in Eq. (A13) is regarded as the convolution of $D_{\mu \alpha}$ and $\left[\partial c_{\mu}^{s} / \partial \theta_{\mu}\right] P_{t}$ and the integration over $\mathbf{r}$ with multiplying $P_{w}$. Since the convolution is expressed as the product in reciprocal space, the summation over $\mathbf{r}$ and $\mathbf{r}^{\prime}$ is converted to the following form:

$$
-\frac{1}{\Delta^{3}} \sum_{w} B_{s w}\left[\frac{1}{V} \sum_{\mathbf{k}} \widetilde{P}_{w}(-\mathbf{k}) \widetilde{D}_{\mu \alpha}(k) \widetilde{G}_{\mu t}(\mathbf{k})\right],
$$

where $\widetilde{D}_{\mu \alpha}=\widetilde{H}_{\mu \alpha}^{V V}-\delta_{\mu t}$ and $\widetilde{G}_{\mu t}$ is the Fourier transform of $\left[\partial c_{\mu}^{s} / \partial \theta_{\mu}\right] P_{t}$. We notice that the basis $P_{w}(\mathbf{r})$ is related to $P_{1}(\mathbf{r})$,

$$
P_{w}(\mathbf{r})=P_{1}\left(\mathbf{r}-\mathbf{R}_{1 w}\right),
$$

where $\mathbf{R}_{1 w}$ is the vector pointing from the $P_{1}$ 's center to the $P_{w}$ 's one. In reciprocal space, this relation is expressed as

$$
\widetilde{P}_{w}(\mathbf{k})=\widetilde{P}_{1}(\mathbf{k}) e^{i \mathbf{k} \cdot \mathbf{R}_{1 w}} .
$$

Inserting the above equation to Eq. (A14), we obtain the Jacobian matrix in a computationally useful form,

$$
\begin{aligned}
J_{\alpha s, \mu t}= & \delta_{\alpha \mu} \delta_{s t}-\frac{1}{\Delta^{3}} \sum_{w} B_{s w} \\
& \times\left[\frac{1}{V} \sum_{\mathbf{k}} \widetilde{P}_{1}(-\mathbf{k})\left\{\widetilde{H}_{\mu \alpha}^{V V}(k)-\delta_{\alpha \mu}\right\} \widetilde{G}_{\mu t}(\mathbf{k}) e^{-i \mathbf{k} \cdot \mathbf{R}_{1 w}}\right] .
\end{aligned}
$$

The double summation is converted to the preparation of $\widetilde{P}_{1}$ 
and $\widetilde{G}_{\mu t}$, the multiplication in reciprocal space, and the backward Fourier transformation.

${ }^{1}$ See, for example, S. Nugent and B. M. Ladanyi, J. Chem. Phys. 120, 874 (2004).

${ }^{2}$ J. Tomasi, B. Mennucci, and R. Cammi, Chem. Rev. (Washington, D.C.) 105, 2999 (2005).

${ }^{3}$ J. Tomasi and M. Persico, Chem. Rev. (Washington, D.C.) 94, 2027 (1994).

${ }^{4}$ C. J. Cramer and D. G. Truhlar, Chem. Rev. (Washington, D.C.) 99, 2161 (1999).

${ }^{5}$ C. G. Gray and K. E. Gubbins, Theory of Molecular Fluids Volume 1: Fundamentals (Oxford University Press, New York, 1984).

${ }^{6}$ L. Blum and A. J. Torruella, J. Chem. Phys. 56, 303 (1972).

${ }^{7}$ L. Blum, J. Chem. Phys. 57, 1862 (1972).

${ }^{8}$ L. Blum, J. Chem. Phys. 58, 3295 (1973).

${ }^{9}$ P. H. Fries and G. N. Patey, J. Chem. Phys. 82, 429 (1985).

${ }^{10}$ J. Richardi, C. Millot, and P. H. Fries, J. Chem. Phys. 110, 1138 (1999).

${ }^{11}$ N. Yoshida and S. Kato, J. Chem. Phys. 113, 4974 (2000).

${ }^{12}$ S. Ten-no, F. Hirata, and S. Kato, J. Chem. Phys. 100, 7443 (1994).

${ }^{13}$ H. Sato, in Molecular Theory of Solvation, edited by F. Hirata (Kluwer Academic, Dordrecht, 2003), pp. 61-99.

${ }^{14}$ H. Sato, F. Hirata, and S. Kato, J. Chem. Phys. 105, 1546 (1996).

${ }^{15}$ D. Beglov and B. Roux, J. Chem. Phys. 103, 360 (1995).

${ }^{16}$ D. Beglov and B. Roux, J. Chem. Phys. 104, 8678 (1996).

${ }^{17}$ M. Ikeguchi and J. Doi, J. Chem. Phys. 103, 5011 (1995).

${ }^{18}$ C. M. Cortis, P. J. Rossky, and R. A. Friesner, J. Chem. Phys. 107, 6400 (1997).

${ }^{19}$ A. Kovalenko, in Molecular Theory of Solvation, edited by F. Hirata (Kluwer Academic, Dordrecht, 2003), pp. 169-276.

${ }^{20}$ A. Kovalenko and F. Hirata, J. Phys. Chem. B 103, 7942 (1999).

${ }^{21}$ A. Kovalenko and F. Hirata, J. Chem. Phys. 110, 10095 (1999).

${ }^{22}$ H. Sato, A. Kovalenko, and F. Hirata, J. Chem. Phys. 112, 9463 (2000).

${ }^{23}$ Q. Du and D. Wei, J. Phys. Chem. B 107, 13463 (2003).

${ }^{24}$ N. Yoshida and F. Hirata, J. Comput. Chem. 27, 453 (2006).

${ }^{25}$ G. N. Chuev and M. V. Fedorov, J. Comput. Chem. 25, 1369 (2004).

${ }^{26}$ G. N. Chuev and M. V. Fedorov, J. Chem. Phys. 120, 1191 (2004).

${ }^{27}$ M. Kawata, C. M. Cortis, and R. A. Friesner, J. Chem. Phys. 108, 4426 (1998).

${ }^{28}$ A. Kovalenko, S. Ten-no, and F. Hirata, J. Comput. Chem. 20, 928 (1999).

${ }^{29}$ M. Kinoshita, Y. Okamoto, and F. Hirata, J. Comput. Chem. 18, 1320 (1997)

${ }^{30}$ M. Kinoshita, in Molecular Theory of Solvation, edited by F. Hirata (Kluwer Academic, Dordrecht, 2003), pp. 101-168.

${ }^{31}$ M. Kinoshita and F. Hirata, J. Chem. Phys. 104, 8807 (1996)

${ }^{32}$ G. P. Morriss and D. MacGowan, Mol. Phys. 58, 745 (1986).
${ }^{33}$ H. J. C. Berendsen, J. P. M. Postma, M. F. van Gunsteren, and J. Hermans, in Intermolecular Forces, edited by B. Pullman (Reidel, Dordrecht, 1981), p. 331.

${ }^{34}$ M. J. Gillan, Mol. Phys. 38, 1781 (1979).

${ }^{35}$ We defined the finite Fourier expansion as $\bar{f}(\mathbf{k})=\int_{V} d \mathbf{r} f(\mathbf{r}) e^{i \mathbf{k} \cdot \mathbf{r}}$ and $f(\mathbf{r})$ $=V^{-1} \sum_{\mathbf{k}} \bar{f}(\mathbf{k}) e^{-i \mathbf{k} \cdot \mathbf{r}}$, where $V$ is the cell volume.

${ }^{36}$ G. J. Martyna and M. E. Tuckerman, J. Chem. Phys. 110, 2810 (1999).

${ }^{37}$ L. Füsti-Molnár and P. Pulay, J. Chem. Phys. 116, 7795 (2002).

${ }^{38}$ L. Füsti-Molnár and P. Pulay, J. Chem. Phys. 117, 7827 (2002).

${ }^{39}$ L. Füsti-Molnár and J. Kong, J. Chem. Phys. 122, 074108 (2005).

${ }^{40}$ M. W. Schmidt, K. K. Baldridge, J. A. Boatz et al., J. Comput. Chem. 14, 1347 (1993).

${ }^{41}$ T. H. Dunning, Jr. and P. J. Hay, in Methods of Electronic Structure Theory, edited by H. F. Schaefer III (Plenum, New York, 1977).

${ }^{42}$ K. Naka, A. Morita, and S. Kato, J. Chem. Phys. 110, 3484 (1999).

${ }^{43}$ W. D. Cornell, P. Cieplak, C. I. Bayly, I. R. Gould, K. M. Merz, Jr., D. M. Ferguson, D. C. Spellmeyer, T. Fox, J. W. Caldwell, and P. A. Kollman, J. Am. Chem. Soc. 117, 5179 (1995).

${ }^{44}$ W. L. Jorgensen and T. B. Nguyen, J. Comput. Chem. 14, 195 (1993).

${ }^{45}$ K. Hirao, Chem. Phys. Lett. 190, 374 (1992).

${ }^{46}$ H. A. Witek, Y.-K. Choe, J. P. Finley, and K. Hirao, J. Comput. Chem. 23, 957 (2002).

${ }^{47}$ M. Dupuis, J. Rys, and H. F. King, J. Chem. Phys. 65, 111 (1976).

${ }^{48}$ J. Rys, M. Dupuis, and H. F. King, J. Comput. Chem. 4, 154 (1983).

${ }^{49}$ A. Ben-Naim and Y. Marcus, J. Chem. Phys. 81, 2016 (1984).

${ }^{50}$ H. Krienke, G. Schmeer, and A. Straßer, J. Mol. Liq. 113, 115 (2004).

${ }^{51}$ C. Amovilli and B. Mennucci, J. Phys. Chem. B 101, 1051 (1997).

${ }^{52}$ L. Sandberg, R. Casemyr, and O. Edholm, J. Phys. Chem. B 106, 7889 (2002).

${ }^{53}$ J. Li, T. Zhu, G. D. Hawkins, P. Winget, D. A. Liotard, C. J. Cramer, and D. G. Truhlar, Theor. Chem. Acc. 103, 9 (1999).

${ }^{54}$ K. Kimura and S. Nagakura, Theor. Chim. Acta 3, 164 (1965).

${ }^{55}$ C. J. Seliskar, O. S. Khalil, and S. P. McGlynn, in Excited States, edited by E. C. Lim (Academic, New York, 1974), vol. 1.

${ }^{56}$ O. S. Khalil, J. L. Meeks, and S. P. McGlynn, Chem. Phys. Lett. 39, 457 (1976).

${ }^{57}$ G. W. King and A. A. G. van Putten, J. Mol. Spectrosc. 42, 514 (1972).

${ }^{58}$ J. Del Bene and H. H. Jaffé, J. Chem. Phys. 49, 1221 (1968).

${ }^{59}$ A. L. Sobolewski and W. Domcke, Chem. Phys. Lett. 250, 428 (1996).

${ }^{60}$ O. Kröhl, K. Malsch, and P. Swiderek, Phys. Chem. Chem. Phys. 2, 947 (2000).

${ }^{61}$ S. Nagakura, M. Kojima, and Y. Maruyama, J. Mol. Spectrosc. 13, 174 (1964).

${ }^{62}$ B. Vidal and J. N. Murrell, Chem. Phys. Lett. 31, 46 (1975).

${ }^{63}$ N. S. Bayliss and E. G. McRae, J. Phys. Chem. 58, 1006 (1954).

${ }^{64}$ H. Labhart and G. Wagnière, Helv. Chim. Acta 46, 1314 (1963). 